NORDITA-2009-21

\title{
String Mass Shifts
}

\author{
Diego Chialva \\ Nordita Institute AlbaNova University Centre, \\ Roslagstullsbacken 23 SE-106 91 Stockholm, Sweden \\ chialva@nordita.org
}

\begin{abstract}
We study closed string one-loop amplitudes in string theory, in particular the average mass shift for states at given mass and NeveuSchwarz charges. Our analysis is based only on well-defined string amplitudes and the exploitation of symmetries and unitarity properties of the torus amplitudes.

We obtain the result $\Delta M^{2}=-g_{s}^{2} M^{2+\frac{3-D}{2}}$ in $D$ space-time dimensions for the average closed string mas-shift $\left(\Delta M^{2}=-g_{s}^{2}\left(M^{2}-\right.\right.$ $\left.Q^{2}\right)^{1+\frac{3-D}{4}}$ for states with non-zero Neveu-Schwarz charges $\left.Q\right)$. An interesting picture of one-loop corrections for the string in non - supersymmetric configurations comes out: the dominant interactions responsible for these corrections are of long-range type (namely gravitational) and it appears that perturbations theory is generally reliable on the spectrum of massive string states.
\end{abstract}




\section{Contents}

1 Introduction $\quad 1$

2 String formula for the one-loop amplitude.

3 Real and imaginary part of the one-loop amplitude 8

3.1 Expansion of the one-loop amplitude . . . . . . . . . . 8

3.2 Integrals: real and imaginary part . . . . . . . . . . . . 11

3.2.1 The imaginary part . . . . . . . . . . . . . . . 14

3.2 .2 The real part . . . . . . . . . . . . . 15

3.3 Coefficients . . . . . . . . . . . . . 16

3.3.1 The finite difference equation . . . . . . . . 16

3.3.2 The solution of the finite difference equation . . . . . . 18

4 The average mass shift for closed string states $\quad 19$

4.1 Definition of the relevant decay rates . . . . . . . . . . . . 20

4.2 Dominant channels of interaction . . . . . . . . . 23

4.3 Computation of the coefficients . . . . . . . . . 26

4.3.1 Gravitons, dilatons, Kalb-Ramond fields, Kaluza-Klein,

Winding modes and Scalars (NS-NS sector) . . . . . . 26

4.3.2 Spinors and gauge fields (R-R sector) . . . . . . . . 27

4.3.3 NS-R and R-NS sectors . . . . . . . . . . . . . 29

4.3 .4 Total . . . . . . . . . . . . . . . . . 29

4.4 The average mass shift . . . . . . . . . . . . . . . 30

5 Discussion and Conclusion 33

6 Acknowledgments

A Computation of the residue $\mathcal{R}_{m}^{r, s}$

B Arguments for suppression due to averaging 37

C Theta functions 38

\section{Introduction}

String theory is a promising candidate for a quantum theory of gravity and all the fundamental interactions. One of its appealing features is that a perturbative expansion is possible and formally well-defined. Nonetheless, 
in fact very few is know in detail, already at the level of one-loop string amplitudes.

Knowledge of one-loop corrections (mass shifts) is of great importance both for fundamental aspects of the theory and for applications in the absence of a protection mechanism against renormalization such as supersymmetry. At the fundamental level it can confirm or question the reliability of the formal expansion in powers of the string coupling, and better shape the region of parameters (coupling) for which it is valid. Applications, on the other hand, are represented for example by investigations of the black holes/string correspondence for non-supersymmetric configurations [1, 2, 3].

The study of one-loop string diagrams has so far mainly focused on some aspects: first of all, on the imaginary part of the diagrams for particular states [4, 5, 6]. Indeed, thanks to the optical theorem the imaginary part has an on-shell definition, which makes the computation, at least in principle, straightforward.

As for the real part, properties such as the lack of UV divergences, unitarity, conformal invariance, ... were those generally investigated [7]. Only a restricted number of works (see [8] and references) have coped with the computation of the actual magnitude of the corrections. In fact those papers have dealt only with particular states (such as those with maximal angular momentum), because of various advantages in the technology needed to discuss them.

General studies on average mass shifts or similar statistical information on the whole range of the string massive spectrum have instead developed some sort of field theory or even semiclassical approximations of the string calculation, but so far none of these attempts has proven fully reliable. In particular they have generally assumed the predominance of gravitational interactions in the self-energy of a string, but without verifying it on welldefined string amplitudes.

The difficulties in coping with the mass shifts of strings are both technical and conceptual. On the one side, they are the consequence of the lack of definition of the theory so far, still bound to an on-shell first-quantized version 1 , and on the other, they are due to the complexity in the computation of well-defined on-shell string amplitudes (see section 2 for a brief discussion).

This paper provides a rigorous study of string mass shifts, and in particular statistical information concerning the average mass shift for the whole spectrum of massive states a a given mass. In fact, we will be able to clarify

\footnotetext{
${ }^{1}$ Studies on the definition and computation of off-shell amplitudes do exist, see for example [9] and references, but the techniques are not well-formalized and most of the results are in the field theory limit $\alpha^{\prime} \rightarrow 0$ and in the bosonic string theory.
} 
some of the statements expressed in the past about string mass shifts.

We will investigate closed string one-loop amplitudes and establish an algorithm to study them efficiently, individuating the imaginary part, the real one, the different asymptotic contributions and finally computing the mass shift for large masses. Fully determining the dominant contribution will turn out to be quite involved, but we will provide arguments and evidence for our conclusions.

Our algorithm is well suited for both pure string states and mixed ones. This is of particular interest, since it allows studying general average properties of string mass shifts. We stress, in any case, that the only ingredients in the algorithm are well defined string off-shell amplitudes.

The paper is so subdivided: in Section 2 we review the string formulas for one-loop amplitudes and summarize some of their features which will be important in the following. In section 3 we re-write the one-loop amplitude in a convenient way by expanding it 2 as a sum of terms constituted by a coefficient and an integral part. We then study these respectively in sections 3.2 and 3.3. We finally apply our techniques to the computation of the average mass shift for states at a certain mass level $N$ and charges $Q^{i}$ in section 4 .

Finally, we comment and conclude.

\section{String formula for the one-loop amplitude.}

Studying the mass shift of fundamental closed strings in perturbative string theory means to compute torus amplitudes with the insertions of two vertex operators representing the string state of interest (see figure 1). Such calculations are difficult to be performed and even defined in string theory for a series of reasons:

- the form of vertex operators for massive states is complicated

- looking for statistical properties means in principle to be able to compute one-loop two-points amplitudes for all possible string states in an ensemble, but only a few vertex operators are explicitly known

- one-loop two-points amplitudes are divergent (due to the presence of an imaginary part), therefore issues of analytical continuation must be carefully studied. This is difficult within String Theory, since in its present status the theory is defined only on-shell.

\footnotetext{
${ }^{2}$ No approximation is involved in this.
} 


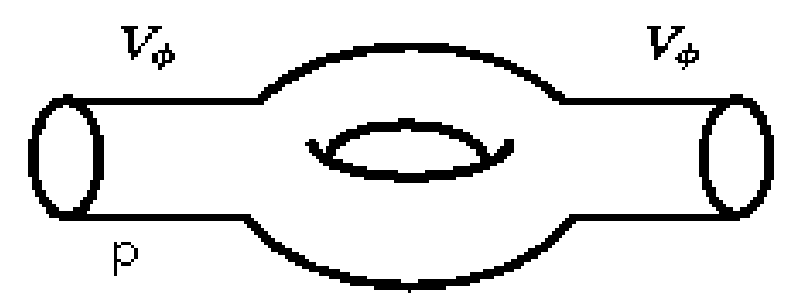

Figure 1: Two-point one-loop amplitude for the state represented by the vertex operator $V_{\phi}$ with four-momentum $p$.

An optimal method for solving (some of) these problems and computing would be factorization [8]: starting from a known four-point amplitude, we can factorize the external legs pairwise and obtain the squared mass shifts for the intermediate states as the residue of the double pole for the center of mass energy. In that case we do not need the detailed knowledge of the form of vertex operators and, as we said above, the squared momentum flowing in the loop is now a continuous variable, allowing analytical continuation.

Unfortunately this approach has a residual unsolved problem: in order to identify mass shifts for the various states we need to know the form of all their couplings with the external legs of the amplitude 3 .

In this work we will take another approach, considering instead welldefined one-loop two-point string amplitudes and extracting from them the string mass shifts for the states of our interest.

Our setup is closed superstring theory 4 with $10-D$ small compactified dimensions 5 (we consider toroidal compactification, for simplicity). The string states taken into account are extended in the uncompactified dimensions (that is they are constituted by excitations of the uncompactified string coordinate operators only), but can possibly wrap or have momentum charges (Neveu-Schwarz charges) along the compact dimensions.

\footnotetext{
${ }^{3}$ For particular states, namely those on the Regge trajectory, which are non-degenerate, the method can be implemented, see 8].

${ }^{4}$ IIA or IIB, even though our techniques could be extended to type I theory.

${ }^{5}$ We use $D$ for indicating the space-time extended dimension, $d=D-1$ for the spatial ones.
} 
We work in a time gauge ([10]), such that the on-shell vertex operators have the form 6 :

$$
V_{\phi}=e^{-i p^{0} X^{0}+i \vec{p} \cdot \vec{X}} V\left(X^{i}, \psi^{i}, S^{\alpha}\right)
$$

where the $S^{\alpha}$ are spin-fields and $i$ runs over the spatial dimensions. For the bosonic string we would have $V_{\phi}=e^{-i p^{0} X^{0}+i \vec{p} \cdot \vec{X}} V\left(X^{i}\right)$.

Furthermore, we choose to work in the center-of-mass reference system, where

$$
p^{\mu}=\left\{p^{0}, \overrightarrow{0},\left\{p_{L}^{j}, p_{R}^{j}\right\}\right\}, \quad j=d+1, d+2, \ldots,
$$

where $\overrightarrow{0}$ gives the momentum spatial components in the extended dimensions and $j$ runs over the compact ones.

To simplify the notation, we consider initial states $\phi$ having only KaluzaKlein or winding charges, not present both at the same time 7 . Therefore, being the vertex operators on-shell,

$$
\left(p^{0}\right)^{2}=N+Q^{2} \equiv N+\sum_{j}\left(p_{L(R)}^{j}\right)^{2}, \quad\left|p_{L}^{j}\right|=\left|p_{R}^{j}\right|
$$

where $M_{0}^{2}=N+Q^{2}$ is the tree-level squared mass.

Note that the excitations present in $V\left(X^{i}, \psi^{i}, S^{\alpha}\right)$ have $i \notin\{d+1, d+$ $2, \ldots, D\}$, because the states we consider are extended only in the uncompactified dimensions. Finally, in our units $\alpha^{\prime}=4$.

The string one-loop on-shell amplitude for a string state represented by such a vertex operator $V_{\phi}$ is 8

$$
\begin{aligned}
i T_{T^{2}}= & i g_{s}^{2} \iiint\left\langle V_{\phi}(0) V_{\phi}(\nu)\right\rangle_{T^{2}} \\
= & i g_{s}^{2} \int d^{2} \tau d^{2} \nu \frac{e^{-4 \pi\left(N+Q^{2}\right) \frac{\operatorname{Im}(\nu)^{2}}{\operatorname{Im}(\tau)}}}{(\operatorname{Im}(\tau))^{\frac{d+1}{2}}} \frac{L\left(d_{c}, d, \tau, \bar{\tau}, \nu, \bar{\nu}\right)}{|\eta(\tau)|^{2(D-2)}}\left|\frac{\theta_{1}(\nu, \tau)}{\theta_{1}^{\prime}(0, \tau)}\right|^{4 N} \\
& \times \mathcal{P}_{\phi}\left(W, \Omega, \partial_{\nu} \Omega, . ., \bar{\Omega}, \partial_{\bar{\nu}} \bar{\Omega}, . .\right) \\
& \times \mathcal{X}_{\phi}(\nu, \tau) \tilde{\mathcal{X}}_{\phi}(\bar{\nu}, \bar{\tau})
\end{aligned}
$$

\footnotetext{
${ }^{6}$ Here and in the following, our vertex operators do not have the usual closed string coupling factor $g_{s}$ carried by the string vertex operators. This is because for clarity we have decided to explicitly show all string coupling factors in the formulas for the amplitudes.

${ }^{7}$ In any case, all the analysis in this work can be repeated for cases where both charges are present at the same time: it is sufficient to 1 ) distinguish the right- and left-moving factors in the amplitude by introducing $N_{R}$ and $N_{L}$ respectively instead than $N, 2$ ) operate two distinct expansions in formula (14) with indexes $s, \tilde{s}$ and 3) modify accordingly the analysis in the rest of this work.

${ }^{8}$ Here and in the following a bar over a quantity indicates its complex conjugate. Also, the overall normalization of the one-loop amplitude has been absorbed in the correlator $\left\langle V_{\phi}(0) V_{\phi}(\nu)\right\rangle_{T^{2}}$ for simplicity of notation.
} 
with

$$
\begin{aligned}
\Omega & =\partial_{\nu}^{2} \ln \left(e^{-\frac{\pi \nu^{2}}{\operatorname{Im}(\tau)}} \theta_{1}(\nu, \tau)\right) \\
W & =\frac{2 \pi}{\operatorname{Im}(\tau)}
\end{aligned}
$$

and it is related to the S-matrix by:

$$
S=11+i T \text {. }
$$

In the above formula, $\mathcal{P}_{\phi}$ is a polynomial of $W, \Omega, \bar{\Omega}$ and their higher derivatives and $\mathcal{X}_{\phi}\left(\tilde{\mathcal{X}}_{\phi}\right)$ is the (anti)holomorphic fermionic part of the amplitude 9 .

All the relevant information regarding the state $|\phi\rangle$, contained in the factor $V\left(X^{i}, \psi^{i}, S^{\alpha}\right)$ of the vertex operator (see formula (10) ), is stored in the quantities $\mathcal{P}_{\phi}, \mathcal{X}_{\phi}, \tilde{\mathcal{X}}_{\phi}$. In particular, the amplitude for mixed string states, represented by a density matrix such as

$$
\rho=\sum_{\phi} c_{\phi}|\phi\rangle\langle\phi|
$$

can be written, using the vertex operator formalism, as the sum over the one-loop two point functions for the physical states $|\phi\rangle$ with the relevant coefficients $c_{\phi}$. The form of the summed up amplitude is therefore analogous to (44), with $\mathcal{P}_{\phi}, \mathcal{X}_{\phi},, \tilde{\mathcal{X}}_{\phi}$ replaced by $\mathcal{P}_{\rho}, \mathcal{X}_{\rho}, \overline{\mathcal{X}}_{\rho}$ depending on $W, \Omega, \bar{\Omega}$ and higher derivatives, as well as the various $\left\{c_{\phi}\right\}$.

If we knew explicitly the form of the vertex operator $V_{\phi}$, then we could fully determine the quantities $\mathcal{P}_{\phi}, \mathcal{X}_{\phi}, \tilde{\mathcal{X}}_{\phi}$ (similarly for mixed states, if we knew the form of the vertex operators for the states entering the definition of the density matrix). We will however show how symmetries and unitarity properties of the torus help in specifying them up to some elements that can be computed directly, even in cases where not all the relevant vertex operators are known (such as for the string average, see section 44).

In formula (4), the contribution from the compactified dimensions is given by $L\left(d_{c}, d, \tau, \bar{\tau}, \nu, \bar{\nu}\right)$, which, assuming for simplicity compactification on a torus, reads

$$
\begin{aligned}
L\left(d_{c}, d, \tau, \bar{\tau}\right)= & \prod_{i=1}^{d_{c}-d} \frac{1}{R_{(i)}} e^{\sum_{n_{i}, w_{i}} 2 \pi i \tau \sum_{i}\left(\frac{n^{i}}{R^{i}}+\frac{w_{i} R^{i}}{4}\right)^{2}-2 \pi i \bar{\tau} \sum_{i}\left(\frac{n^{i}}{R^{i}}-\frac{w_{i} R^{i}}{4}\right)^{2}} \\
& \times e^{-4 \pi i \nu \sum_{i} p_{L}^{i}\left(\frac{n^{i}}{R^{i}}+\frac{w_{i} R^{i}}{4}\right)+4 \pi i \bar{\nu} \sum_{i} p_{R}^{i}\left(\frac{n^{i}}{R^{i}}-\frac{w^{i} R^{i}}{4}\right)},
\end{aligned}
$$

\footnotetext{
${ }^{9}$ If we were to study the bosonic string theory, the formula to use would be pretty similar, with a different $\mathcal{P}_{\phi}$ and no fermionic $\mathcal{X}_{\phi}\left(\tilde{\mathcal{X}}_{\phi}\right)$.
} 
where $d_{c}$ is the critical (spatial) dimension, that is 9 for the superstring 10 .

The coordinate $\tau=\tau_{1}+i \tau_{2}$ represents the torus moduli and is therefore integrated over the complex plane after dividing out the gauge transformations that preserve the metric (see [11, volume 1, chapter 5), namely, on the torus, modular transformations. This translates, as we will discuss more at length in the following, in a subdivision of the complex plane in several fundamental regions and the integration is restricted to one of these. It is customary to choose the one defined as

$$
F=\left\{\tau \in \mathbb{C}:|\tau| \geq 1,\left|\tau_{1}\right| \leq \frac{1}{2}\right\}
$$

On the other hand, $\nu=\nu_{1}+i \nu_{2}$ represents the modulus related to the position of one of the vertex operators (the other one is fixed by the conformal Killing vectors on the torus, see [11]) and is integrated over a region

$$
0 \leq \nu_{2} \leq \tau_{2}, \quad\left|\nu_{1}\right| \leq \frac{1}{2}
$$

Finally, a brief note about the singularities of the one-loop amplitude. It is well-known that there are no UV singularities because they would arise from the region of integration where $\tau_{2} \rightarrow 0$, which is eliminated because of modular invariance (see (11)). The remaining singularities arise all from IR behavior.

In particular the dangerous limit is represented by $\nu \rightarrow 0$, that is when the two insertion points of the vertex operators approach each other. The divergence is a tadpole which signals a modification of the background. Nonetheless in most supersymmetric backgrounds (such as the one we have here) these IR divergences actually vanish, while in the non-supersymmetric case they are understood in terms of the Fischler-Susskind mechanism [12].

Having reviewed the general features of one-loop string amplitudes, we now turn to a detailed study of their real and imaginary part:11. We will proceed in the following way:

- rewrite the amplitude as a sum over different terms, each constituted of a coefficient part and an integral over the moduli $\tau, \nu$

\footnotetext{
${ }^{10}$ It would be 25 for the bosonic theory.

${ }^{11}$ Recall that due to unitarity, the imaginary part of the one-loop S-matrix is related to the sum over the final products of the squared modulus of the tree-level S-matrix for the decays of the initial state:

$$
T_{T^{2}, \phi}-\bar{T}_{T^{2}, \phi}=i \sum_{\phi^{\prime}, \zeta}\left|T_{\text {tree }, \phi, \phi^{\prime}, \zeta}\right|^{2} .
$$


- focus on the integral part distinguishing the real and imaginary parts

- focus on the coefficient part

- compute the real part in a specific case: the average for large fixed mass and fixed charges.

\section{Real and imaginary part of the one-loop amplitude}

In this section we are going to obtain the main formulas we will use in the computation of the string one-loop diagrams.

\subsection{Expansion of the one-loop amplitude}

We will now analyze formula (4) and suitably expand its integrand. In the following we do not distinguish between pure and mixed states, since what we will say is valid in both case 12 .

To proceed, we need to distinguish the holomorphic and anti-holomorphic part of the integrand. The factorization in these two parts is not exact, due to the presence of the mixed derivative term $W$, but the integrand can be expanded in powers of $W$ and every term will factorize independently. Therefore, we write

$$
\begin{aligned}
\mathcal{P}\left(W, \Omega, \partial_{\nu} \Omega, . ., \bar{\Omega}, \partial_{\bar{\nu}} \bar{\Omega}, . .\right) & = \\
& =\sum_{s} W^{s}(\tau) \mathcal{M}_{s}\left(\Omega, \partial_{\nu} \Omega, \ldots, \bar{\Omega}, \partial_{\bar{\nu}} \bar{\Omega}, \ldots\right) \\
& =\sum_{s} W^{s}(\tau) \sum_{u, \tilde{u}} \Omega^{u} \bar{\Omega}^{\tilde{u}} \mathcal{U}_{s, u}\left(\partial_{\nu} \Omega, \ldots\right) \overline{\mathcal{U}}_{s, \tilde{u}}\left(\partial_{\bar{\nu}} \bar{\Omega}, \ldots\right) .
\end{aligned}
$$

Using Newton's binomial

$$
\begin{aligned}
\Omega^{u} & =\left(\partial_{\nu}^{2} \log \theta_{1}-\frac{2 \pi}{\tau_{2}}\right)^{u} \\
& =\sum_{f=0}^{u}\left(\begin{array}{l}
u \\
f
\end{array}\right)\left(\partial_{\nu}^{2} \log \theta_{1}\right)^{u-f}\left(\frac{2 \pi}{\tau_{2}}\right)^{f}(-1)^{f}
\end{aligned}
$$

and defining

$$
r \equiv f+s, \quad \tilde{r} \equiv \tilde{f}+s
$$

\footnotetext{
${ }^{12}$ The notation will vary accordingly: we will neglect the suffixes in $\mathcal{P}_{\phi / \rho}, \mathcal{X}_{\phi / \rho}$ and simply write $\mathcal{P}, \mathcal{X}$ instead.
} 
we obtain

$$
\begin{aligned}
& \mathcal{P}\left(W, \Omega, \partial_{\nu} \Omega, . ., \bar{\Omega}, \partial_{\bar{\nu}} \bar{\Omega}, . .\right)= \\
& \sum_{s, r, \tilde{r}} \frac{1}{\tau_{2}^{r+\tilde{r}-s}} \mathcal{D}_{s}^{r}\left(\partial^{2} \log \theta_{1}, \partial^{3} \log \theta_{1}, \ldots\right) \overline{\mathcal{D}}_{s}^{\tilde{r}}\left(\bar{\partial}^{2} \log \bar{\theta}_{1}, \bar{\partial}^{3} \log \bar{\theta}_{1}, \ldots\right)
\end{aligned}
$$

where we have defined

$$
\begin{aligned}
& \mathcal{D}_{s}^{r}\left(\partial^{2} \log \theta_{1}, \partial^{3} \log \theta_{1}, \ldots\right)= \\
& \sum_{u}(2 \pi)^{\frac{s}{2}}\left(\begin{array}{c}
u \\
r-s
\end{array}\right)\left(\partial_{\nu}^{2} \log \theta_{1}\right)^{u-r+s}(-2 \pi)^{r-s} \mathcal{U}_{s, u}\left(\partial_{\nu} \Omega, \ldots\right) .
\end{aligned}
$$

Finally, we consider

$$
\left(2 \pi i \frac{\theta_{1}(\nu, \tau)}{\theta_{1}^{\prime}(0, \tau)}\right)^{2 N} \mathcal{D}_{s}^{r}\left(\partial^{2} \log \theta_{1}, \partial^{3} \log \theta_{1}, \ldots\right) \mathcal{X}_{\phi}(\nu, \tau) \equiv \mathcal{F}^{r, s}(\nu, \tau)
$$

and expand it in powers of

$$
v \equiv e^{2 i \pi \nu} \quad w \equiv e^{2 i \pi \tau} .
$$

The coefficients of the power series in $v$ are

$$
\frac{1}{2 \pi i} \oint \frac{d v}{v} v^{m+N} \mathcal{F}^{r, s}=\chi_{m}^{r, s}(w)
$$

The expansion in powers of $v$ is valid inside an annulus between $v=1$ and $v=w$ (which are singular points, recall also (12)). Remember, anyhow, the discussion about the singularities in string one-loop amplitudes after (12): we found that the singularity at $v=1(\nu=0)$ is actually not present for supersymmetric backgrounds (our case) 13 . Therefore the integrand of the one-loop amplitude has actually a zero at $v=1$.

We will show in section 3.3.1 that the coefficients (22) obey a finite difference equation,which will allow us to obtain their general form and the dependence on the quantum numbers identifying the state under consideration. Before doing that, we complete the analysis of the one-loop amplitude formula.

\footnotetext{
${ }^{13}$ It is instead understood in terms of the Fischler-Susskind mechanism when supersymmetry is absent.
} 
We further expand 14 as

$$
\chi_{m}^{r, s}(w)=\sum_{p} \chi_{m, p}^{r, s} w^{p}
$$

Note that $m+p \geq 0, \forall m, p 15$ This will have a physical interpretation in (32).

After expanding in the same way the anti-holomorphic part in power of $\bar{v}, \bar{w}$, we can finally write (4) as

$$
\begin{aligned}
T_{T^{2}}=g_{s}^{2} \int \frac{d^{2} \tau}{\tau_{2}^{\frac{d+1}{2}+r+\tilde{r}-s}} \int d^{2} \nu e^{-4\left(N+Q^{2}\right) \pi \frac{\nu_{2}^{2}}{\tau_{2}}} \sum_{s, r, \tilde{r}} \sum_{p, m, \tilde{p}, \tilde{m}} \chi_{-m, p}^{r, s} \tilde{\chi}_{-\tilde{m}, \tilde{p}}^{\tilde{r}, s} \\
\times e^{2 \pi i p \tau+2 \pi i m \nu} e^{-2 \pi i \tilde{p} \bar{\tau}-2 \pi i \tilde{m} \bar{\nu}} \\
\times \prod_{i=1}^{d_{c}-d} \frac{1}{R_{(i)}} \sum_{n^{i}, w^{i}} e^{\sum_{n_{i}, w_{i}} i \tau\left(\frac{n^{i}}{R^{i}}+\frac{w^{i} R^{i}}{4}\right)^{2}-i \bar{\tau}\left(\frac{n^{i}}{R^{i}}-\frac{w^{i} R^{i}}{4}\right)^{2}} \\
\times e^{-4 \pi i \nu \sum_{i} p_{L}^{i}\left(\frac{n^{i}}{R^{i}}+\frac{w^{i} R^{i}}{4}\right)+4 \pi i \bar{\nu} \sum_{i} p_{R}^{i}\left(\frac{n^{i}}{R^{i}}-\frac{w^{i} R^{i}}{4}\right)} .
\end{aligned}
$$

\footnotetext{
${ }^{14}$ Note that the coefficient of the series in powers of $v, w, \bar{v}, \bar{w}$ are real. This can be understood as follows. Let us start with the bosonic part. The term $\left(2 \pi i \frac{\theta_{1}(\nu, \tau)}{\theta_{1}^{\prime}(0, \tau)}\right)^{2 N}$ has clearly an expansion with real coefficients. The polynomial $\mathcal{P}$ depends on derivatives of the Green functions. The state-vertex relation is $\alpha_{-n}^{\mu} \rightarrow i \frac{\partial_{n}^{n} X^{\mu}}{(n-1) !}$ and the analog for rightmoving quantities. By Wick's theorem, every term of $\mathcal{P}$ is given by a product of two-point correlators of the form $\left\langle i \partial_{\nu}^{n} i \partial_{\nu}^{m} G\right\rangle$ and analogues with anti-holomorphic derivatives as well. The Green function on the torus for the scalar coordinates is $G=-\frac{\alpha^{\prime}}{2} \log \left|2 \pi i \frac{\theta_{1}(\nu, \tau)}{\theta_{1}^{\prime}(0, \tau)}\right|^{2}+$ $\alpha^{\prime} \pi \frac{(\nu-\bar{\nu})^{2}}{4 \tau_{2}}$ which has an expansion of the form we are considering with real coefficients (the background charge part will be acted upon by the derivatives and become a constant term). Every $\partial_{\nu}$ or $\partial_{\bar{\nu}}$ acting on $G$ brings down a factor $i$. But every term in the polynomial $\mathcal{P}$ has an even number of derivatives because of the level matching condition. Therefore the coefficient of the expansion are real. The same is valid for the antiholomorphic part.

The fact that the fermionic contribution has also an expansion in terms of real coefficients follows from the same considerations, for example taking a bosonized form for the world-sheet fermions and considering level matching as well as conservation of fermion number.

${ }^{15}$ This can be proven by observing that the torus amplitude can be divided into an invariant contribution under $\nu \rightarrow \nu+\tau$, see section 3.3.1 and a part which transform with periodicities related to the Neveu-Schwarz charges of the state. The terms we are expanding here are part of the invariant contribution. Furthermore, the torus integrand has a finite limit for $\tau_{2} \rightarrow \infty$ (that is $w \rightarrow 0$ ). Therefore for every expansion term of the form $\chi_{m, p} w^{p} v^{m}$ under $\nu \rightarrow \nu+\tau$ we have $w^{p} v^{m} \rightarrow w^{p+m} v^{m}$ and since the amplitude must be finite for $w \rightarrow 0$, then $m+p \geq 0$.
} 
We see that the amplitude is now written as a sum of different terms constituted by some coefficient factors $\chi_{-m, p}^{r, s} \tilde{\chi}_{-\tilde{m}, \tilde{p}}^{\tilde{r}, s}$ and some integral factors

$$
\begin{aligned}
& H_{m, p, \tilde{m}, \tilde{p},\left\{n^{i}, w^{i}\right\}}= \int \frac{d^{2} \tau}{\tau_{2}^{\frac{d+1}{2}+r+\tilde{r}-s}} e^{2 i \pi \tau_{1}\left(p-\tilde{p}+\sum_{i=1}^{6} w_{i} n_{i}\right)-2 \pi \tau_{2}\left(p+\tilde{p}+\sum_{i=1}\left(\frac{2 n_{i}^{2}}{R_{i}^{2}}+\frac{w_{i}^{2} R_{i}^{2}}{8}\right)\right)} \\
& \int d^{2} \nu e^{-4\left(N+Q^{2}\right) \pi \frac{\nu_{2}^{2}}{\tau_{2}}+2 i \pi \nu_{1}(m-\tilde{m})-2 \pi \nu_{2}(m+\tilde{m}-2 N)} \\
& \times e^{-4 \pi i \nu \sum_{i} p_{L}^{i}\left(\frac{n^{i}}{R^{i}}+\frac{w^{i} R^{i}}{4}\right)+4 \pi i \bar{\nu} \sum_{i} p_{R}^{i}\left(\frac{n^{i}}{R^{i}}-\frac{w^{i} R^{i}}{4}\right)}
\end{aligned}
$$

Written in a compact way, it reads:

$$
T_{T^{2}}=g_{s}^{2} \prod_{i=1}^{d_{c}-d} \frac{1}{R_{(i)}} \sum_{n^{i}, w^{i}} \sum_{s, r, \tilde{r}} \sum_{p, m, \tilde{p}, \tilde{m}} \chi_{-m, p}^{r, s} \tilde{\chi}_{-\tilde{m}, \tilde{p}}^{\tilde{r}, s} H_{m, p, \tilde{m}, \tilde{p},\left\{n^{i}, w^{i}\right\}}
$$

The aim of the rest of this work is to determine the coefficient $\left(\chi_{-m, p}^{r, s} \tilde{\chi}_{-\tilde{m}, \tilde{p}}^{\tilde{r}, s}\right)$ and integral $\left(H_{m, p, \tilde{m}, \tilde{p},\left\{n^{i}, w^{i}\right\}}\right)$ factors and finally compute the one-loop amplitude (26). We turn now to study these factors separately and in details.

\subsection{Integrals: real and imaginary part}

We start with the study of the integrals in (25). Performing the integration over $\nu_{1}$, we find

$$
m=\tilde{m}+\sum_{i}\left(n^{i} \mathcal{W}^{i}+w^{i} \mathcal{N}^{i}\right)
$$

where we have written

$$
p_{L(R)}^{i}=\frac{\mathcal{N}_{i}}{R_{(i)}} \pm \frac{\mathcal{W}_{i} R_{(i)}}{4} .
$$

However, recall that we limit ourselves to the case where the string state does not have both non-zero Kaluza-Klein and winding modes at the same time in a compact dimension. This implies $\left|p_{L}^{i}\right|=\left|p_{R}^{i}\right|$ and we define $Q^{i}=p_{L}^{i}$.

In order to discuss the real and imaginary parts arising from the integrals it is convenient to reduce them to a form which is pretty similar to the one a field theory one-loop diagram would have in Schwinger representation. To do that, we change variables as $\nu_{2} \rightarrow \tau_{2} \nu_{2}$ and we find (we suppress indexes on $H$ to neaten formulas)

$$
\begin{aligned}
H= & \int_{F} \frac{d^{2} \tau}{\tau_{2}^{\frac{d+1}{2}+r+\tilde{r}-s-1}} \int_{0}^{1} d \nu_{2} e^{2 i \pi \tau_{1}\left(p-\tilde{p}+\sum_{i=1}^{6} w_{i} n_{i}\right)} \\
& \times e^{-4\left(N+Q^{2}\right) \pi \tau_{2} A\left(\nu_{2}\right)}
\end{aligned}
$$


where we have defined (here $i$ runs over the compactified dimensions)

$$
\begin{aligned}
A\left(\nu_{2}\right)= & \nu_{2}^{2}+\frac{1}{N+Q^{2}}\left(m-N-2 \sum_{i} q_{i} Q^{i}\right) \nu_{2} \\
& +\frac{1}{N+Q^{2}}\left(\frac{p+\tilde{p}-\sum_{i} n^{i} w^{i}}{2}+\sum_{i} q_{i}^{2}\right)
\end{aligned}
$$

with

$$
q_{i}=\left(\frac{n_{i}}{R_{(i)}}+\frac{w_{i} R_{(i)}}{4}\right) .
$$

Comparing this with a field theory one-loop diagram for a coupling $\phi \varphi_{1} \varphi_{2}$, we find

$$
\begin{aligned}
M_{1}^{2} & =\frac{p+\tilde{p}}{2}+\sum_{i}\left(\frac{n_{i}^{2}}{R_{(i)}^{2}}+\frac{w_{i}^{2} R_{(i)}^{2}}{16}\right) \\
M_{1}^{2}-M_{2}^{2} & =-m+2 \sum_{i}\left(\frac{n_{i}}{R_{(i)}}+\frac{w_{i} R_{(i)}}{4}\right) Q^{i}-\sum_{i} Q_{i}^{2},
\end{aligned}
$$

where $M_{1}, M_{2}$ are the masses of the states (also virtual ones) circulating in the loops.

The function $A\left(\nu_{2}\right)$ is a parabola. For $m>N$ it is always positive and therefore there is no imaginary part for the integral. However when $m<N$ and for certain $p, \tilde{p}, n_{i}, w_{i}, A\left(\nu_{2}\right)$ is negative over a certain range of $\nu_{2}$, which results in an imaginary part for the string self-energy. In that case the integral as it stands is not well-defined and we have to use analytical continuation for it.

Differently from field theory, we do not know the form of the amplitude for generic squared momenta $p^{2}$, where $p^{\mu}$ is the momentum of the external lines in the diagram in figure 1. In fact, we can define it only for specific discrete points $p^{2}=-\left(N+Q^{2}\right)$, corresponding to the masses of on-shell string states, because only in that case the vertex operators in the initial formula (41) are correctly defined. Furthermore, note that off-shell string amplitudes are generally quite different form their on-shell limit/counterpart (see [13] and references therein). We therefore cannot trust an analytical continuation to negative $\left(N+Q^{2}\right)$.

What we can do is instead to analytically continue the variable $\tau$ (and therefore $\nu$ ). This is indeed the correct procedure, since the integrand in $H$ is defined over continuous regions of $\tau, \nu$. Also, see [4], recall that physically the string world-sheet propagator is defined as

$$
\Delta=\int_{0}^{+\infty} d \tau_{2} \int_{0}^{2 \pi} d \tau_{1} \frac{1}{[\text { Invariances }]} e^{-i \tau_{2}\left(L_{0}+\tilde{L}_{0}-i \epsilon\right)-i \tau_{1}\left(L_{0}-\tilde{L}_{0}\right)}
$$




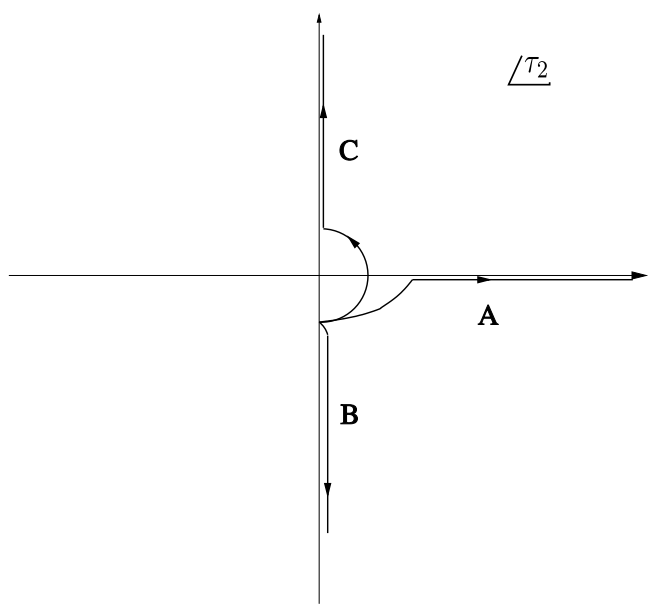

Figure 2: The analytical continuation for the integration over the modulus $\tau$.

and we usually consider its Euclidean continuation $\tau_{2} \rightarrow-i \tau_{2}$ (from $A$ to $B$, in figure 2). However, this continuation is possible only if the eigenvalues of $\left(L_{0}+\tilde{L}_{0}-i \epsilon\right)$ are positive defined. This is not the case when the system is unstable and we can have decay, and this leads to the aforementioned imaginary parts.

The correct analytical continuation is, in that case, $\tau_{2} \rightarrow i \tau_{2}$ (from $A$ to $C$ in figure 2). In performing it 16 , we obtain an imaginary part and a real part. Indeed the new path $C$ of integration for $\tau_{2}$ can be divided into a contribution from the imaginary axis and a semicircle shrinking around $\tau=0$. The imaginary part comes from the integration around the semicircle, as was discussed and obtained in [5, 4].

The real part comes from the integration of $\tau_{2}$ along the axis. In terms of $\tau$, the integration is over the complex plane, dividing out the invariances of the metric, namely modular invariance. As we already mentioned this leads to the definition of fundamental regions of integration.

Note that in the usual operatorial formulation of torus amplitudes in string theory [18, the restriction of the integration region over a fundamental domain is sometimes inferred by checking invariance of the one-loop amplitude once it is already analytically continued to $\tau_{2} \rightarrow-i \tau_{2}$, but the amplitude is formally divergent (due to the imaginary part)!

The true physical reason for the restriction of the integration region is

\footnotetext{
${ }^{16}$ Note that the total amplitude, when analytically continued remains convergent since the eta and theta functions are still convergent (only a finite number of terms in their expansion in powers of $w=e^{2 \pi i \tau}$ will be affected by the continuation, namely those powers with exponent $0<n<N$ only).
} 
instead the necessity of dividing out the gauge invariances of the torus metric (see Polchinski volume 1, chapter 5)

$$
d s^{2}=\left|d \sigma_{1}^{2}+\tau d \sigma_{2}^{2}\right|
$$

and this must be taken into account by any analytical continuation.

We will now present first the formulas for the imaginary part, and then those for the real one.

\subsubsection{The imaginary part}

For specific values of $m(<N), p, \tilde{p}$ and $\left\{q^{i}\right\}$, we can write (30), as

$$
A\left(\nu_{2}\right)=\left(\nu_{2}-y_{-}\right)\left(\nu_{2}-y_{+}\right)
$$

with two real root $y_{+}, y_{-}$given by ( $i$ runs over the compactified dimensions)

$$
\begin{aligned}
y_{ \pm}= & -\frac{1}{2\left(N+Q^{2}\right)}\left(m-N-2 \sum_{i} q_{i} Q^{i}\right) \\
& \pm \sqrt{\left(\frac{m-N-2 \sum_{i} q_{i} Q^{i}}{2\left(N+Q^{2}\right)}\right)^{2}-\frac{1}{\left(N+Q^{2}\right)}\left(\frac{p+\tilde{p}-\sum_{i} n^{i} w^{i}}{2}+\sum_{i} q_{i}^{2}\right)} .
\end{aligned}
$$

Here $n, p, n_{i}, w_{i}$ run only over values that make the square root real.

From (29), after the analytical continuation and integration over $\tau_{1}$,

$\operatorname{Im}(H)=\int_{y_{-}}^{y_{+}} d \nu_{2} \frac{\sin \left(\pi\left(p-\tilde{p}+\sum_{i} n_{i} w_{i}\right)\right.}{\pi\left(p-\tilde{p}+\sum_{i} n_{i} w_{i}\right)} \pi \frac{\left(4\left(N+Q^{2}\right) \pi\left|A\left(\nu_{2}\right)\right|\right)^{\left(\frac{d+1}{2}+r+\tilde{r}-s-2\right)}}{\Gamma\left(\left(\frac{d+1}{2}+r+\tilde{r}-s-1\right)\right)}$,

where $\nu_{2}$ is integrated over the range where $A\left(\nu_{2}\right)$ is negative (see (35)).

As we can see, the imaginary part is non-zero only for

$$
p-\tilde{p}+\sum_{i \in \operatorname{comp}} n_{i} w_{i}=0
$$

which ensures that the emitted string states satisfy the level matching condition.

Integrating over $\nu_{2}$ and inserting back the result in (24), we obtain

$$
\operatorname{Im}\left(T_{T^{2}}\right)=g_{s}^{2} \prod_{i=1}^{d_{c}-d} \frac{1}{R_{(i)}} \sum_{\left\{n^{i}, w^{i}\right\}} \sum_{s, r, \tilde{r}} \sum_{m, p} \chi_{-m, p}^{r, s} \tilde{\chi}_{-\tilde{m}, \tilde{p}}^{\tilde{r}, s} \frac{\pi}{2 \sqrt{N+Q^{2}}} \frac{\left(4 \pi \varpi^{2}\right)^{\frac{d+1}{2}+r+\tilde{r}-s-\frac{3}{2}}}{\Gamma\left(\frac{d}{2}+r+\tilde{r}-s\right)}
$$


where we have defined ( $i$ runs over the compactified dimensions)

$$
\begin{aligned}
\varpi & \equiv \sqrt{N+Q^{2}} \frac{y_{+}-y_{-}}{2} \\
& =\sqrt{\left(\frac{m-N-2 \sum_{i} q_{i} Q^{i}}{2 \sqrt{N+Q^{2}}}\right)^{2}-\left(\frac{p+\tilde{p}-\sum_{i} n^{i} w^{i}}{2}+\sum_{i} q_{i}^{2}\right)}
\end{aligned}
$$

and $\tilde{p}$ is given by (38).

The result (39) is analogous to those, for example, in [6].

\subsubsection{The real part}

We must consider, defining it by analytical continuation where necessary,

$$
\begin{aligned}
H= & \int_{F} \frac{d^{2} \tau}{\tau_{2}^{\frac{d+1}{2}+r+\tilde{r}-s}} e^{2 i \pi \tau_{1}(p-\tilde{p})-2 \pi \tau_{2}\left(p+\tilde{p}-\sum_{i} n^{i} w^{i}+2 \sum_{i} q_{i}^{2}\right)} \\
& \int d^{2} \nu e^{-4\left(N+Q^{2}\right) \pi \frac{\nu_{2}^{2}}{\tau_{2}}-4 \pi \nu_{2}\left(m-N-2 \sum_{i} q_{i} Q^{i}\right)} .
\end{aligned}
$$

The integral does not exist in finite form. In order to estimate correctly its value, we need to know better the asymptotes of the integrand in the various regions of integration. These will depend on the values of $m, p, \tilde{p}, \ldots$ and of course on $N, Q$. It is therefore convenient to discuss them once we will have a more precise knowledge of the other ingredients of the one-loop amplitude (the expansion coefficients (23)) that will enable us to individuate the dominating contributions in terms of $m, p, \tilde{p}, \ldots$.

In any case, by inspecting (41) we can start arguing how the integral factor would affect, and suppress, certain contributions to the total sum over $p, \tilde{p}, m, n^{i}, w^{i}$. The dominant contributions should give the correct asymptotic behavior of the whole amplitude.

We observe that terms with large $p, \tilde{p}, q^{2}$ would be generally suppressed by the exponential in the first line of (41) and force $\tau_{2}$ to the lower limit of its integration region, which in turn would suppress the contribution due to the exponential in the second line (in particular due to the first term in the exponential). Analogously, contributions with $m \gg N$ will be similarly suppressed.

Note that, due to the identification (32), the channel(s) where $p, \tilde{p}=0$ correspond to the case where at least one of the two states running in the 
loop is massless or a pure Kaluza-Klein/winding mode17. That massless interactions (namely the gravitational one) dominate the mass shift for very massive string is expected on general reasons $18([2,3])$ and will be further discussed in section 4.2 .

Evidently, we need to check if the more or less suppression due to the integral factors in the amplitude is compensated or not by the behavior of the coefficient factors. Therefore our argument so far is not sufficient to determine the favored channels of interaction.

We will now indeed turn to the study of the coefficients in the expansion of the one-loop amplitude.

\subsection{Coefficients}

In this section we are going to obtain the coefficients of the expansion of the one-loop amplitude (26), as defined in section 3.1, formulas (22, 23).

\subsubsection{The finite difference equation}

We will exploit the transformation properties of the one-loop amplitudes under some symmetry transformations. This will enable us to write a system of finite difference equations that the coefficients (22) must obey. By solving it we will obtain the form of the coefficients. The discussion goes in parallel for both $\chi_{m}^{r, s}$ and $\tilde{\chi}_{\tilde{m}}^{\tilde{r}, s}$, therefore we will treat in details the first ones.

Beside the contour $\mathcal{C}$ we used in the definition (22), we consider two different contours $19 \mathcal{C}^{\prime}, \mathcal{C}^{\prime \prime}$ (see figure 3), related by the transformations $v^{\prime}=$ $v w^{-1}, v^{\prime \prime}=w v^{-1}$.

Let us understand how the integrand of the one-loop amplitude (4) behaves under the transformations that relate the various loops integrations. Knowledge of some properties of the integrand in (4) will be useful at this

\footnotetext{
${ }^{17}$ The condition we give is for $M_{1}^{2}=0$. The condition for $M_{2}^{2}=0$ is $m=-\frac{p+\tilde{p}}{2}$, but since the amplitude is invariant under $M_{1}^{2} \leftrightarrow M_{2}^{2}$ (one can check it comes from the invariance under $\nu \leftrightarrow \tau-\nu$ of the periodical -quantum- contribution to the torus amplitude, see section 3.3.11, we need only to treat the case $p=\tilde{p}=0$, which will turn out to be simpler to deal with, given the formulas (52) for the coefficients.

${ }^{18}$ The rationale behind this idea is that the gravitational interaction grows with the mass and therefore becomes the dominant one for very massive states.

${ }^{19}$ The idea of exploiting specific transformations laws in order to obtain general equations constraining string amplitudes, when expanded in suitable power series, was first used in 14 on partial inclusive tree-level amplitudes. Here we apply it to one-loop amplitudes, modifying it where useful. Ours analysis of the equations and their solution is different from that in [14].
} 


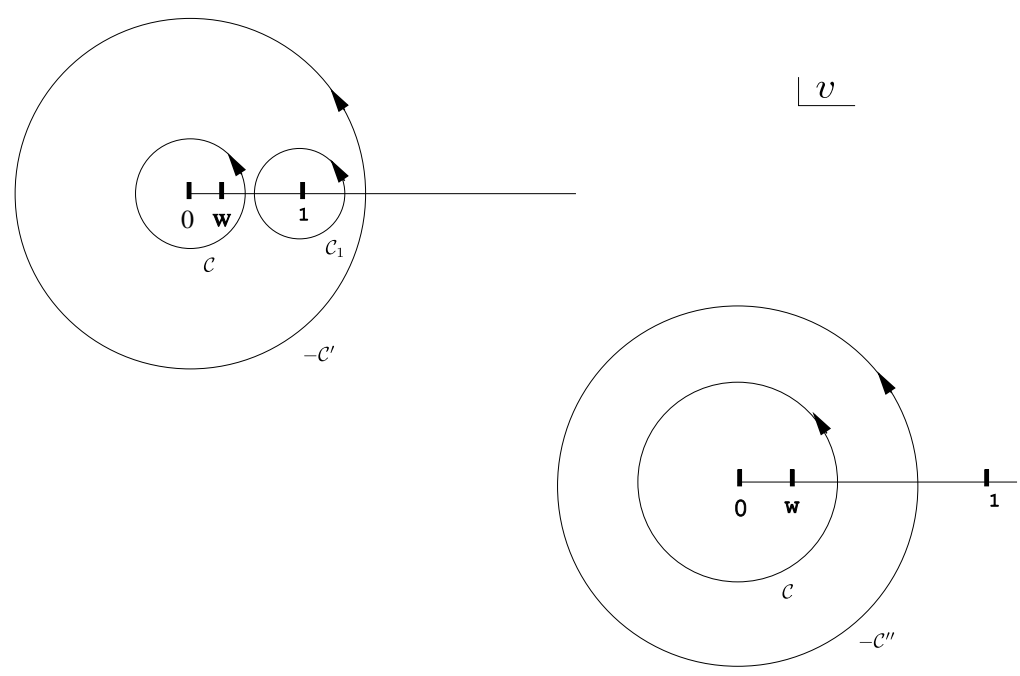

Figure 3: The contours and relevant deformations involved in the derivation of the finite difference equation for the expansion coefficients of the one-loop amplitude.

point. In particular, under the torus periodicities

$$
\nu \rightarrow \nu \pm 1, \quad \nu \rightarrow \nu \pm \tau
$$

the compactified coordinates transform as

$$
\begin{aligned}
& X^{i}(\nu \pm 1)=X^{i}(\nu)+2 \pi R_{(i)} w^{i} \\
& X^{i}(\nu \pm \tau)=X^{i}(\nu)+2 \pi R_{(i)} l^{i}
\end{aligned}
$$

where $l^{i}$ is the dual (in the sense of Poisson resummation) of the KaluzaKlein number $n^{i}$. The path integral can be computed as usual by writing $X^{i}$ as a classical solution with the correct periodicity plus a quantum piece with periodic boundary conditions.

Since our states are not excited in the compactified dimensions, the quantum (periodic) part is the only one that enters in $\mathcal{F}^{r, s}(\nu, \tau)$, defined in (20). Invariance under (42) of the total periodic contribution to the torus amplitude implies that it transforms as

$$
\mathcal{F}^{r, s}(\nu \pm \tau, \tau)=e^{\mp i 4 N \pi \nu} e^{-i 2 N \pi \tau} \mathcal{F}^{r, s}(\nu, \tau) .
$$

By opportunely deforming the various contours, picking up the residues at the singular points $v=0,1$, we find

$$
\begin{aligned}
\frac{1}{2 \pi i} \oint_{\mathcal{C}^{\prime}} \frac{d v^{\prime}}{v^{\prime}} v^{\prime m+N} \mathcal{F}^{r, s}\left(v^{\prime}, w\right) & =\frac{1}{2 \pi i} \oint_{\mathcal{C}^{\prime}} \frac{d v}{v} \frac{v^{m+3 N}}{w^{m+2 N}} \mathcal{F}^{r, s}(v, w) \\
& =\frac{1}{2 \pi i} \oint_{\mathcal{C}} \frac{d v}{v} v^{m+N} \mathcal{F}^{r, s}(v, w)+\mathcal{R}_{m}^{r, s}
\end{aligned}
$$


with

$$
\mathcal{R}_{m}^{r, s}=\frac{1}{2 \pi i} \oint_{\mathcal{C}_{1}} \frac{d v}{v} v^{m+N} \mathcal{F}^{r, s}(v, w),
$$

where $\mathcal{C}_{1}$ is a circuit around $v=1$.

Similarly 20

$$
\begin{aligned}
\frac{1}{2 \pi i} \oint_{\mathcal{C}^{\prime \prime}} \frac{d v^{\prime \prime}}{v^{\prime \prime}} v^{\prime \prime m+N} \mathcal{F}^{r, s}\left(v^{\prime \prime}, w\right) & =\frac{1}{2 \pi i} \oint_{\mathcal{C}^{\prime \prime}} \frac{d v}{v} \frac{v^{N-m}}{w^{-m}} \mathcal{F}^{r, s}(v, w) \\
& =\frac{1}{2 \pi i} \oint_{\mathcal{C}} \frac{d v}{v} v^{m+N} \mathcal{F}^{r, s}(v, w) .
\end{aligned}
$$

We compute the residue $\mathcal{R}_{m}^{r, s}$ in the appendix $\mathrm{A}$, with the result

$$
\mathcal{R}_{m}^{r, s}=0 \text {. }
$$

Using the definition (22), equations (46, 48) read

$$
\left\{\begin{array}{l}
\chi_{m+2 N}^{r, s}(w)=w^{m+2 N} \chi_{m}^{r, s}(w) \\
\chi_{-m}^{r, s}(w)=w^{-m} \chi_{m}^{r, s}(w) .
\end{array}\right.
$$

\subsubsection{The solution of the finite difference equation}

We will now solve the system (50). First, we note that the integrand of the torus amplitude has a finite result for $w \rightarrow 0\left(\tau_{2} \rightarrow \infty\right)$. This implies that the expansion in series of $w$ for all coefficients $\chi_{m}^{r, s}(w)$, for both positive and negative $m$, can have only positive or null powers. Then, consider the last equation of (50): for $m>0$, being $w^{-m}$ a negative power of $w$, it must be

$$
\chi_{m}^{r, s}(w) \sim a_{m} w^{m}
$$

The general solution of (50) is

$$
\chi_{ \pm m}^{r, s}(w)=w^{A N(A \pm 1)+\eta\left(A+\frac{1 \pm 1}{2}\right)} \chi_{-\eta}^{r, s}(w)
$$

with

$$
0 \leq \eta \leq 2 N, \quad m=2 A N+\eta .
$$

The solutions depend on some unknown coefficients $\chi_{-\eta}^{r, s}, 0 \leq \eta \leq 2 N$. They contain the information on the specific state (pure or mixed) whose one-loop

\footnotetext{
${ }^{20}$ Here we also used the invariance of $\mathcal{F}^{r, s}(\nu, \tau)$ under under $\nu \rightarrow-\nu$, due to the fact that the whole amplitude is invariant under the modular transformations $\nu \rightarrow \frac{\nu}{c \tau+d}, \quad \tau \rightarrow$ $\frac{a \tau+b}{c \tau+d}, \quad a d-c b=1$ and we can choose $a=d=-1, b=c=0$.
} 
two-point function we analyze. Not all of them are independent, in fact using (50), we see that only the coefficients with $0 \leq \eta \leq N$ are such.

The system (50) by itself is not enough to determine them, but these coefficients (with one exception) are those which participate in the imaginary part of the amplitude. The only exception is the terms $\eta=N$, for which the amplitude does not have an imaginary part. This is the only coefficient we cannot fully determine. We will deal with the contribution $\eta=M$ in more details in section 4.4.

We can then compute the coefficients with $0 \leq \eta \leq N-1$ from the tree-level amplitudes for the decays of our initial state into two string states. Unfortunately this again would in principle require the knowledge of the form of all string vertex operators, which we do not possess.

We turn now to the discussion of the specific case we are interested in: the average closed string mass shift. We will see that it is possible to estimate what are the dominant contributions to the one-loop self-energy and compute exactly the relevant coefficients and integral factors in that case.

\section{The average mass shift for closed string states}

The aim of this section is to obtain the average mass shift for closed string states at three-level squared mass $M_{0}^{2}=N+Q^{2}$ for fixed $N$ and $Q$. To the reader's convenience, let us summarize here our achievements so far and let us anticipate what we are going to do now.

In the previous sections, we have studied in details the one-loop string amplitude, individuating its imaginary and real parts. The amplitude has been written in terms of a sum over coefficients and integral terms. Formulas for the integrals have been given, both for the imaginary and the real part. Formulas for the coefficients have been given as well, in terms of a subset of such coefficients that strictly depend on the state under consideration. On the other hand, we have proven in section 3.2 that this remaining subset of coefficients (a part from one) can actually be determined by comparison to three-level amplitudes (decay rates).

We are going now to

- define the relevant amplitudes and decay rates in order to obtain the coefficients which are still unknown in (52).

- obtain the asymptotic behavior of the whole on-loop amplitude for large initial $N=M_{0}^{2}-Q^{2}$ by studying the different kinds of contributions and individuating the asymptotically dominant ones. 


\subsection{Definition of the relevant decay rates}

We define here the tree-level computations useful to obtain the remaining unknown expansion coefficients in (52) for the one-loop average string amplitude. They have the form:

$$
\Gamma=g_{s}^{2} \mathcal{P} \sigma_{L}^{\mathrm{av}} \times \sigma_{R}^{\mathrm{av}}
$$

where

- $\mathcal{P}$ is the phase space, including the sum over the Neveu-Schwarz charges of the final states and the normalization factor for the amplitude

$$
\sigma_{L}^{\mathrm{av}}=\frac{1}{\sqrt{G_{c}(N)}} \sum_{\phi_{\left.\right|_{N}}} \sum_{\phi^{\prime}, \zeta}\left|\left\langle\phi_{L}^{\prime}\left|V_{\zeta, L}\right| \phi_{L}\right\rangle\right|^{2}
$$

is the sum over the final states $21 \phi^{\prime}, \zeta$ of the modulus square of the decay amplitudes for the state(s) $\phi$, averaged over physical states $\phi$ at mass level $N=N_{R}=N_{L}$ carrying fixed charges $Q^{i}=Q_{L}^{i},\left|Q_{R}^{i}\right|=\left|Q_{L}^{i}\right| . \quad \sigma_{R}$ is obtained substituting to the left-moving quantities the right-moving ones. $G_{c}(N)$ is the degeneracy of closed string states at mass-level $N$.

In order to compare the sum over tree-level decays with the imaginary part of the one-loop amplitude, it is convenient to reorganize the sum over states $\phi^{\prime}, \zeta$ as a double sum over states at a given mass-level $N^{\prime}, \aleph$ and all possible mass-levels:

$$
\sum_{\zeta}=\sum_{\aleph} \sum_{\zeta_{\aleph}} \quad \sum_{\phi^{\prime}}=\sum_{N^{\prime}} \sum_{\phi_{N_{N^{\prime}}}^{\prime}}
$$

and define

$$
\sigma_{L}^{\aleph, N^{\prime}} \equiv \sum_{\zeta_{\left.\right|_{\aleph}}, \phi_{\left.\right|_{N}}} \frac{1}{\sqrt{G_{c}(N)}} \frac{1}{2 \pi i} \oint \frac{d z^{\prime}}{z^{\prime N^{\prime}+1}}\left\langle\phi_{L}\left|V_{\zeta, L}^{\dagger}(1) z^{\prime \hat{N}_{L}} V_{\zeta, L}(1)\right| \phi_{L}\right\rangle .
$$

having used a mass-projector ([15])

$$
\rho_{N^{\prime}}=\frac{1}{2 \pi i} \oint \frac{d z^{\prime}}{z^{\prime N^{\prime}+1}} z^{\prime \hat{N}_{L}}
$$

such that

$$
\sum_{\phi_{\left.\right|_{N^{\prime}} ^{\prime}}}\left|\phi_{L}^{\prime}\right\rangle\left\langle\phi_{L}^{\prime}\left|=\sum_{\phi_{\text {physical }}^{\prime}} \rho_{N^{\prime}}\right| \phi_{L}^{\prime}\right\rangle\left\langle\phi_{L}^{\prime}\right|
$$

\footnotetext{
${ }^{21}$ Our notation for closed string states here is $|\phi\rangle=\left|\phi_{L}\right\rangle\left|\phi_{R}\right\rangle$.
} 
The average case can be discussed in terms of a density matrix

$$
\rho_{\mathrm{av}}=\sum_{\phi_{\left.\right|_{\text {physical }}}} c_{\phi}|\phi\rangle\langle\phi| .
$$

defined by

$$
\begin{cases}c_{\phi} \equiv G_{c}(N) & \text { for } \phi \text { at mass-level } N \\ c_{\phi} \equiv 0 & \text { for } \phi \text { not at mass-level } N\end{cases}
$$

In our case, as we said, it is also $N_{R}=N_{L}=N$ (see (3)) ).

We can see then that the average is just a particular case of a more general scenario and that our approach can be extended to other cases and used for obtaining other statistical informations using different density matrices representing different mixed states.

Let us concentrate now on the average case only. Its density matrix can be re-written as $\rho_{\text {av }}=\rho_{N} \tilde{\rho}_{N}$ with

$$
\rho_{N}=\frac{1}{\sqrt{G_{c}(N)}} \frac{1}{2 \pi i} \oint \frac{d x}{x^{N+1}} x^{\hat{N}_{L}},
$$

and we obtain

$$
\begin{aligned}
\sigma_{L}^{\aleph, N^{\prime}} & =\sum_{\zeta_{\aleph}, \phi_{\left.\right|_{N}}} \frac{1}{\sqrt{G_{c}(N)}} \frac{1}{2 \pi i} \oint \frac{d z^{\prime}}{z^{\prime N^{\prime}+1}}\left\langle\phi_{L}\left|V_{\zeta, L}^{\dagger}(1) z^{\hat{N}_{L}} V_{\zeta, L}(1)\right| \phi_{L}\right\rangle \\
& =\frac{1}{\sqrt{G_{c}(N)}} \frac{1}{(2 \pi i)^{2}} \sum_{\zeta_{\left.\right|_{\aleph}}} \oint \frac{d x}{x^{N+1}} \oint \frac{d z^{\prime}}{z^{\prime N^{\prime}+1}} \operatorname{tr}\left[V_{\zeta, L}^{\dagger}(1) V_{\zeta, L}\left(z^{\prime}\right)\left(x z^{\prime}\right)^{\hat{N}_{L}}\right] \\
& =\frac{1}{\sqrt{G_{c}(N)}} \frac{1}{(2 \pi i)^{2}} \sum_{\zeta_{\aleph}} \oint \frac{d z}{z^{N+1}} \oint \frac{d z^{\prime}}{z^{\prime}} z^{\prime N-N^{\prime}} \operatorname{tr}\left[V_{\zeta, L}^{\dagger}(1) V_{\zeta, L}\left(z^{\prime}\right) z^{\hat{N}_{L}}\right]
\end{aligned}
$$

where in the last line we have changed variables to

$$
z \equiv x z^{\prime}
$$

Here $\hat{N}_{L}$ is the left-moving mass-level operator 22 . We can write $\sigma_{R}$ in the same way using $\hat{N}_{R}$.

The last line of formula (63) is our main formula: $\sigma_{L(R)}^{\aleph, N^{\prime}}$ written in this way can be computed in operator formalism in the same way as the one-loop two

\footnotetext{
${ }^{22}$ We will always distinguish an operator from its value by mean of $\mathrm{a}^{\wedge}$.
} 
point function for on-shell vertex operators $V_{\zeta}^{\dagger}, V_{\zeta}$, just without integrating over the zero modes, without spin-structure one-loop signs and projecting at the end on some mass-level. This ensures that only physical states enter in the trace and therefore that these on-shell amplitudes are well-defined in string theory.

Let us fix clearly the notation for future use (repeated index is summed):

- the initial state has momentum $p^{\mu}$, mass-level $N$, Neveu-Schwarz charges $Q^{i}=Q_{L}^{i},\left|Q_{R}^{i}\right|=\left|Q_{L}^{i}\right|$, from (3) and tree-level squared mass $M_{0}^{2}=$ $N+Q^{2}, Q^{2}=Q^{i} Q_{i}$,

- the emitted state $\zeta$ has momentum $k$, mass-level $\aleph$, charges $q^{i}=q_{L}^{i}$ (and $q_{R}^{i}$ ) and tree-level squared mass $M_{2}^{2}=\aleph+q^{2}, q^{2}=q^{i} q_{i}$,

- the other final state has momentum $p^{\prime}$, mass-level $N^{\prime}$, charges $Q^{\prime i}=Q_{L}^{\prime i}$ (and $\left.Q_{R}^{\prime i}\right)$ and tree-level squared mass $M_{1}^{2}=N^{\prime}+Q^{\prime 2}, Q^{\prime 2}=Q^{\prime i} Q_{i}^{\prime}$.

The phase space then reads

$\mathcal{P}=c \frac{2 \pi^{\frac{d}{2}}}{\Gamma\left(\frac{d}{2}\right)} \prod_{i=1}^{d c-d} \frac{1}{R_{(i)}} \sum_{\left\{q_{i}\right\}} \frac{1}{N+Q^{2}} \sqrt{\left(\frac{N-N^{\prime}+\aleph+2 q^{i} Q_{i}}{2 \sqrt{N+Q^{2}}}\right)^{2}-\aleph-\sum_{i} q^{i} q_{i}}$,

where $c$ is the overall normalization, and so we can write

$$
\begin{aligned}
\Gamma= & c g_{s}^{2} \frac{2 \pi^{\frac{d}{2}}}{\Gamma\left(\frac{d}{2}\right)} \prod_{i=1}^{d_{c}-d} \frac{1}{R_{(i)}} \sum_{\left\{q_{i}\right\}, \aleph, N^{\prime}} \sqrt{\left(\frac{N-N^{\prime}+\aleph+2 q^{i} Q_{i}}{2 \sqrt{N+Q^{2}}}\right)^{2}-\aleph-\sum_{i} q^{i} q_{i}} \\
& \times \frac{1}{N+Q^{2}} \sigma_{L}^{\aleph, N^{\prime}} \sigma_{R}^{\aleph, N^{\prime}}
\end{aligned}
$$

We can compare this with the one-loop result for the imaginary part, given in (39, 40).

By using

$$
\frac{4 \pi}{M_{0}} \operatorname{Im}\left(T_{T^{2}}\right)=\Gamma
$$

and we obtain

$$
\sum_{r, \tilde{r}, s} \frac{4^{r+\tilde{r}-s}}{\Gamma\left(\frac{d}{2}+r+\tilde{r}-s\right)} \chi_{-m, p}^{r, s} \tilde{\chi}_{-m, p}^{r, s}=c \frac{2^{3-D}}{\pi \Gamma\left(\frac{d}{2}\right)} \sigma_{R}^{p, m+p} \sigma_{L}^{p, m+p}
$$

where we have used (38) in the case $q_{R}^{i}=q_{L}^{i}$ and (32). 


\subsection{Dominant channels of interaction}

We will try now to argue which, among the possible contributions to the one-loop amplitude, for different $p, \tilde{p}, m, \ldots$, are the dominant ones. These should provide us with the asymptotically correct result for the self-energy.

By looking at (60, 61), an apparent advantage seems to come from the fact that the integrand of the one-loop amplitude for the average case (44) will be weighted by a factor ${ }^{23} G_{c}(N)^{-1}$, where $G_{c}(N)$ is the string degeneracy at level $N$. This would strongly suppress the coefficients of many contributions (see (68)) by a factor $\sim e^{-2 \pi \sqrt{N(d-1)}}$ for large $N$. Non-suppressed contributions are those for which this factor is compensated. However, simple arguments can show that this can in fact happen, because of the summation over all decay channels. These arguments are presented in the appendix $\mathrm{B}$ for what concerns the contribution with non-zero imaginary part.

At the same time, these same remarks exclude that coefficient factors are exponentially enhanced.

On the other hand, as we already said, different considerations point at individuating the dominating contribution to the mass shift in those channels of interactions where at least one of the two states running in the loop is massless or possibly a pure Kaluza-Klein/winding mode.

We call these massless interactions, while the term massive will represent those for which both states running in the loop have non-zero mass level, that is they are massive form a ten dimensional point of view.

On general ground, gravitational interactions (where one of the states running in the loop is a graviton, dilaton, Kalb-Ramond field or in general a superposition of them) are supposed to dominate the self-energy of (very) massive strings since the interaction grows with the mass $([2,3])$.

Another argument for this comes from the direct evaluation and estimate of the asymptotic dependence for large initial mass $M$ of the contributions coming from massless and massive channels. Our expression for the one-loop amplitude (26) allows to easily distinguish the two. We will now discuss the latter and then the supposedly dominant massless channels.

It is difficult to determine exactly the contribution of the total of the massive channels, but we can estimate it, when the massive states running in the loop have $1 \ll M_{1,2}<M$. Indeed, from (68) the relevant coefficient factors in those cases can be obtained from the semi-inclusive decay rate $M \rightarrow M_{1}+M_{2}$. We can estimate the latter in the following way.

A closed string can decay in two massive strings only when two points

\footnotetext{
${ }^{23}$ It will indeed enter the terms $\mathcal{P} \rho_{\mathrm{av}}, \mathcal{X}_{\rho_{\mathrm{av}}}, \overline{\mathcal{X}}_{\rho_{\mathrm{av}}}$ in the amplitude (see section 2), which depend on the density matrix coefficients $c_{\phi}$.
} 
on the string get in contact. The first point can be placed everywhere on the string, and therefore, for a highly massive string behaving like a random walk 1, the probability of decay is directly proportional to the (average) length $N^{\frac{1}{4}}$ of the string. For $M_{1,2} \gg 1$ also the final decay products can be considered as random walks of length $\aleph^{\frac{1}{4}}, N^{\prime \frac{1}{4}}$. Then, the probability for two points at a distance $\min \left(\aleph^{\frac{1}{4}}, N^{\prime \frac{1}{4}}\right)$ to meet is directly proportional to the relative volumes $\left(\frac{N}{\aleph N^{\prime}}\right)^{\frac{D-1}{4}}$ in $d$ spatial dimensions.

Therefore (we use a sloppy notation here)

$$
\sum_{r, \tilde{r}, s} \chi^{r, s} \tilde{\chi}^{\tilde{r}, s} \sim M \Gamma_{M, M_{1}, M_{2}} \sim N^{\frac{1}{4}}\left(\frac{N}{\aleph N^{\prime}}\right)^{\frac{D-1}{4}} .
$$

If we write $N^{\prime}=x N, \aleph \sim(1-x) N$, then

$$
\sum \chi \tilde{\chi} \approx N^{\frac{2-D}{4}}\left(x-x^{2}\right)^{\frac{1-D}{4}} .
$$

We see that the coefficient factors are maximized when either $N^{\prime}$ or $\aleph$ are small, that is when nearly in the massless case.

At the same time, as we have already discussed at the end of section 3.2 , generically all massive channels are suppressed by the integral factor(s) in the amplitude (see (41) for $m, p, \tilde{p} \neq 0$ ). In particular off-shell massive states will be strongly exponentially suppressed by the integrals.

Therefore the only possibly non-negligible contributions, compared to those of the massless channels that we will discuss in a moment (see (81)) appear to occur when one of the states running in the loop is nearly massless. We will consider them a (possibly small) correction to the result given by computing the asymptotic behavior of the massless channels, which will give us the correct asymptotes for the whole amplitude.

We will give further evidence for the suppression of channels other than the massless ones in section 4.4. We will also show that indeed the dominating contribution comes from the gravitational and not other forms of long distance interaction (provided by the other string massless states). We will also see that generally Kaluza-Klein and winding modes are suppressed, and only the lowest ones can be sufficiently excited.

We are aware that the conclusion that the total contribution of massive channels of interactions is really asymptotically subdominant with respect to the massless ones' does not come from a complete and exhaustive calculation, but we believe we have provided some more arguments supporting this, beyond the physical intuition regarding the dominance of gravitational

\footnotetext{
${ }^{24}$ We use the notation introduced in the previous section.
} 
interactions for very large masses. However, even adopting a more conservative and minimal point of view, we will be able at least to obtain the correct corrections due to the massless channels, therefore verifying previous approximated result in the literature, which assumed the predominance of massless (gravitational) interactions from the outset.

Looking back at (32), we see that we have massless or pure KaluzaKlein/winding modes circulating in the loop when 25

$$
p=\tilde{p}=0,
$$

which means that their contribution is given by the term $w^{0}$ in the power series of $\chi_{m}^{r, s}(w)$.

Therefore, taking the limit $w \rightarrow 0$, we find 26

$$
\begin{cases}\chi_{m}^{r, s}(w) \rightarrow 0 & \text { for } m>0 \text { and } m<-2 N \\ \chi_{-m}^{r, s}(w) \rightarrow \text { unknown } & \text { for } 0 \leq m \leq N \\ \chi_{-m}^{r, s}(w)=\chi_{m-2 N}^{r, s}(w) & \text { for } N<m \leq 2 N\end{cases}
$$

using (50, 51, 52). The same occurs for $\tilde{\chi}_{\tilde{m}}^{\tilde{r}, s}$.

The unknown terms are those we will focus on in the following. Since they contribute to the imaginary part (a part from $\chi_{-N}^{r, s}, \tilde{\chi}_{-N}^{\tilde{r}, s}$ which we will consider later on), as we said we can obtain them form a tree-level computation by using formula (68).

In particular, we will compute

$$
\Gamma=g_{s}^{2} \sum_{N^{\prime}} \mathcal{P} \sigma_{L}^{0, N^{\prime}} \sigma_{R}^{0, N^{\prime}}
$$

Performing the sum over all states $\zeta$ at mass level $\aleph=0$ means to compute the quantity

$$
\begin{aligned}
\sigma_{L}^{0, N^{\prime}} \times \sigma_{R}^{0, N^{\prime}} & =\left(\sigma_{L}^{g, N^{\prime}} \sigma_{R}^{g, N^{\prime}}+\sigma_{L}^{s, N^{\prime}} \sigma_{R}^{s, N^{\prime}}+\sigma_{L}^{g, N^{\prime}} \sigma_{R}^{s, N^{\prime}}+\sigma_{R}^{g, N^{\prime}} \sigma_{L}^{s}\right) \\
& =\left(\sigma_{L}^{g, N^{\prime}}+\sigma_{L}^{s, N^{\prime}}\right) \times\left(\sigma_{R}^{g, N^{\prime}}+\sigma_{R}^{s, N^{\prime}}\right),
\end{aligned}
$$

where $\sigma_{L}^{g, N^{\prime}} \sigma_{R}^{g, N^{\prime}}$ comes from the contribution of the NS-NS part of the massless spectrum, $\sigma_{L}^{s, N^{\prime}} \sigma_{R}^{s, N^{\prime}}$ from the R-R part and $\sigma_{L}^{g, N^{\prime}} \sigma_{R}^{s, N^{\prime}}+\sigma_{R}^{s, N^{\prime}} \sigma_{L}^{g, N^{\prime}}$ from the NS-R and R-NS parts.

\footnotetext{
${ }^{25}$ See also note 17

${ }^{26}$ See also appendix $\$$ for a discussion of this result.
} 


\subsection{Computation of the coefficients}

We will now compute in details the decay rate (73). Special care must be given to the issues of normalization: writing the decay rate as $(73,774)$, we must ensure that the various contributions contain the correct relative normalization factors and also that the overall normalization for the total decay rate is correct.

This issue is solved in string theory by computing typical tree-level amplitudes and comparing the results to well-known field theory results. This procedure specifies the normalization of the vertex operators and the amplitude. We will follow these rules, using the conventions in 11. However, eventually, at our level of accuracy, constant factors of order one will be neglected.

\subsubsection{Gravitons, dilatons, Kalb-Ramond fields, Kaluza-Klein, Wind- ing modes and Scalars (NS-NS sector)}

In this case the relevant on-shell vertex operator $V_{\zeta}$ is given by ${ }^{27}$ (restoring $\alpha^{\prime}$ for a moment)

$V_{g}(k, z)=\frac{2}{\alpha^{\prime}} e^{i k \cdot X+i q \cdot X}\left(\partial X^{\mu}-i \frac{\alpha^{\prime}}{2} \psi^{\mu} k \cdot \psi\right)\left(\bar{\partial} X^{\nu}-i \frac{\alpha^{\prime}}{2} \tilde{\psi}^{\mu} k \cdot \tilde{\psi}\right) \xi_{\mu \nu}, \quad k^{2}+q^{2}=0$

which, for $\mu, \nu \in\{0, \ldots, d\}$, is (a superposition of) the graviton, dilaton, Kalb-Ramond field as well as their D-dimensional massive Kaluza-Klein or winding versions (when there are compactified dimensions).

From (63), it is found 28

$$
\begin{gathered}
\sigma_{L}^{g, N^{\prime}}=\sum_{\xi} \frac{\xi \cdot \xi\left(N-N^{\prime}\right)}{\sqrt{G_{c}(N)}} \frac{1}{2 \pi i} \oint \frac{d z}{z^{N^{\prime}+1}} \frac{f(z)^{2-D}}{1-z^{N-N^{\prime}}}\left(z^{-\frac{D-2}{16}} g_{3}(z)^{D-2}\right. \\
\left.-z^{-\frac{D-2}{16}} g_{4}(z)^{D-2}+g_{2}(z)^{D-2}\right)
\end{gathered}
$$

where

$$
\begin{gathered}
f(z)=\prod_{n=1}^{\infty}\left(1-z^{n}\right) \\
g_{3}(z)=\prod_{r=\frac{1}{2}}^{\infty}\left(1+z^{r}\right), \quad g_{4}(z)=\prod_{r=\frac{1}{2}}^{\infty}\left(1-z^{r}\right) \quad g_{2}(z)=\prod_{r=0}^{\infty}\left(1+z^{r}\right) .
\end{gathered}
$$

\footnotetext{
${ }^{27}$ Note also that $k^{\sigma} \xi_{\sigma \nu}+q^{j} \xi_{j \nu}=0$.

${ }^{28}$ We have formally written $\xi_{\mu \nu}=\xi_{\mu} \tilde{\xi}_{\nu}$.
} 
$\sigma_{R}$ is obtained from $\sigma_{L}$ substituting left-moving quantities with right-moving ones. The sum over the various decay products includes a sum over the polarizations as

$$
\sum_{\xi} \xi \cdot \xi=D-2
$$

As found in [15, 16], the loop integral over $z$ has a large contribution when $N^{\prime} \sim N$, yielding

$$
\sigma_{L}^{g, N^{\prime}} \sim\left(N-N^{\prime}\right) \frac{e^{-\pi \sqrt{D-2}\left(\frac{N-N^{\prime}}{2 \sqrt{N}}\right)}}{1-e^{-\pi \sqrt{D-2}\left(\frac{N-N^{\prime}}{2 \sqrt{N}}\right)}}
$$

Expanding:

$$
\sigma_{L}^{g} \sim \frac{\sqrt{N}}{\pi \sqrt{D-2}}
$$

Moreover, from (65, 40) we see that for $m=N^{\prime} \sim N$ only small values of the compact momenta and windings $\left\{q^{i}\right\}$ can be excited. For $m \ll N$ and $p, \tilde{p}$ small, modes with higher values for the $\left\{q^{i}\right\}$ can be excited, but such contributions are suppressed by a factor $e^{-\sqrt{N}+m} \sim e^{-\sqrt{N}}$ from (76).

The contribution form the $D$-dimensional scalars and vectors (having polarization tensor with one or both indexes in the compactified dimensions) are subdominant, since in that case either $\sigma_{L}^{N^{\prime}}$ or $\sigma_{R}^{N^{\prime}}$ or both go like $\sim N-N^{\prime}$, $N^{\prime} \sim N$.

\subsubsection{Spinors and gauge fields ( $R-R$ sector)}

Amplitudes with Ramond-Ramond states are generally difficult to compute within string theory, and the result we present here is novel.

One of the complication we have to face concerns the choice of pictures for the vertex operators which must compensate for the superghost charge anomaly. Therefore, we must consider 29

$$
\sigma_{L}^{s, N^{\prime}}=\frac{1}{\sqrt{G_{c}(N)}} \frac{1}{(2 \pi i)^{2}} \sum_{s} \oint \frac{d z}{z^{N+1}} \oint \frac{d z^{\prime}}{z^{\prime}} z^{\prime N-N^{\prime}} \operatorname{tr}\left[V_{-\frac{1}{2}}(1) V_{-\frac{1}{2}}\left(z^{\prime}\right) z^{\hat{N}_{L}}\right]
$$

\footnotetext{
${ }^{29}$ In the following formulas of this section the index $\pm \frac{1}{2}$ for the vertex operators indicates the picture the operators are written in.
} 
where the on-shell physical vertex operators (left-moving part) are

$$
\begin{aligned}
V_{-\frac{1}{2}}(z, p) & =\frac{\alpha^{\prime \frac{1}{4}}}{\sqrt{2}} \bar{u}_{\dot{\alpha}} S^{\dot{\alpha}}(z) e^{-\frac{\phi}{2}(z)} e^{i k \cdot X(z)+i q \cdot X(z)} \\
V_{\frac{1}{2}}(z, p) & =\left(\frac{2}{\alpha^{\prime}}\right)^{\frac{1}{2}}\left(\frac{i \partial X^{\mu}(z)}{\sqrt{2}}+\frac{\alpha^{\prime}}{2} \frac{p \cdot \psi}{4} \psi^{\mu}\right) \bar{u}_{\dot{\alpha}} \gamma_{\mu \beta} S^{\beta}(z) e^{\frac{\phi}{2}(z)} e^{i k \cdot X(z)+i q \cdot X(z)} \\
k^{2} & +q^{2}=0 .
\end{aligned}
$$

Here

- $\phi$ is the bosonized ghost field

- $S^{\dot{\beta}(\beta)}(z)$ is the spin field

- $u^{\dot{\beta}(\beta)}$ is a spinor wave function.

The right-moving part of the vertex operator is obtained by substituting $\bar{\partial}, \tilde{\psi}, \ldots$ to the analogous quantities here.

The spin field is in a representation of $S O(9,1)$ as in [17]: it is given by a product of spin field for $S O(2)$. Again, using a formula such as (82) allows us to use the same approach as a one-loop amplitude, of course without spin structure one loop phases, therefore only physical states enter the amplitude.

The fact that the spin fields are written as tensor product of $S O(2)$ spin fields make it straightforward to study the cases where the number of extended dimensions $D$ is even. We will therefore limit ourselves to that case 30 .

Before proceeding, it is useful and interesting to observe the Lorentz structures originating from (82). It is straightforward to see that the only non-zero contribution comes from

$$
\operatorname{tr}\left[\bar{u}_{\dot{\alpha}} S^{\dot{\alpha}}(1) e^{-\frac{\phi}{2}(1)} e^{-i k X(1)} \partial X^{\mu}\left(z^{\prime}\right) \bar{u}_{\dot{\alpha}} \gamma_{\mu \beta} S^{\beta}\left(z^{\prime}\right) e^{\frac{\phi}{2}\left(z^{\prime}\right)} e^{i k X\left(z^{\prime}\right)}\right]
$$

whose Lorentz structure is simply

$$
\bar{u} \not p u
$$

which, after using the polarization sum 31

$$
\sum_{s} \bar{u}^{s} u^{s}=\not k+\not l
$$

\footnotetext{
${ }^{30}$ It would be possible to study odd extended dimensions, for example in the light-cone gauge, but at the price of decomposing their spinor fields $S^{a}$ in subgroups representation, which is an effort not worth doing for the present analysis.

${ }^{31}$ The sum over the various decay products (spinors with various internal indices, as seen form $D$ extended dimensions) gives the same result as summing over the ten dimensional polarizations.
} 
leads to a factor

$$
8\left(-\omega E+q^{i} Q_{i}\right)=8\left(N-N^{\prime}\right) .
$$

We therefore obtain (for our definition of the theta functions, see the appendix (C)

$$
\begin{aligned}
\sigma_{L}^{s, N^{\prime}}=\frac{8}{\left(N-N^{\prime}\right)} & \frac{1}{G_{c}(N)} \oint \frac{d z}{(2 \pi i)^{2}} \oint \frac{d z^{\prime}}{z^{N+1}} \frac{f(z)^{\frac{3(2-D)}{2}}}{z^{\prime N^{\prime}+1}}\left(\frac{\theta_{1}^{\prime}(0, z)}{2 \pi i \theta_{1}\left(z^{\prime}, z\right)}\right)^{\frac{D-2}{8}} \\
& \times\left(\theta_{3}^{\frac{D-2}{2}}\left(\sqrt{z^{\prime}}, z\right)-\theta_{4}^{\frac{D-2}{2}}\left(\sqrt{z^{\prime}}, z\right)+\theta_{2}^{\frac{D-2}{2}}\left(\sqrt{z^{\prime}}, z\right)\right)
\end{aligned}
$$

It is difficult at this point to give a final closed formula for this (leftmoving) quantity, since computing the loop integral over $v$ is not straightforward in $D \neq 10$ (remember $D$ is the number of extended dimensions). For $D=10$, in any case, using theta functions identities, we obtain:

$$
\begin{aligned}
\sigma_{L}^{s, N^{\prime}} & =\frac{8\left(N-N^{\prime}\right)}{G_{c}(N)} \frac{1}{(2 \pi i)^{2}} \oint \frac{d z}{z^{N+1}} \oint \frac{d z^{\prime}}{z^{N^{\prime}+1}} \frac{\theta_{2}^{4}(0, z)}{f(z)^{12}} \frac{\theta_{1}^{\prime}(0, z) \theta_{2}\left(z^{\prime}, z\right)}{2 \pi i \theta_{2}(0, z) \theta_{1}\left(z^{\prime}, z\right)} \\
& =\frac{8\left(N-N^{\prime}\right)}{\sqrt{G_{c}(N)}} \frac{1}{2 \pi i} \oint \frac{d z}{z^{N^{\prime}+1}} \frac{f(z)^{8}}{1+z^{N-N^{\prime}}} g_{2}(z)^{8}
\end{aligned}
$$

where $f(w), g(w)$ has been defined in (77).

We see that $\sigma_{R}^{s}$ has a dominant contribution for $N^{\prime} \sim N$, as $\sigma_{R}^{g}$ had, but this time:

$$
\sigma_{L}^{s, N^{\prime}} \sim N-N^{\prime}
$$

We expect similar results in any dimension $D$.

\subsubsection{NS-R and R-NS sectors}

The formula for the emission of gravitinos is given by $\sigma_{R}^{g, N^{\prime}} \sigma_{L}^{s, N^{\prime}}+\sigma_{L}^{s, N^{\prime}} \sigma_{R}^{g, N^{\prime}}$ with

$$
\begin{array}{ll}
\sigma_{L}^{g, N^{\prime}} & \text { given by (76) } \\
\sigma_{L}^{s, N^{\prime}} & \text { given by (89) }
\end{array}
$$

and the correspondent left-moving quantities.

\subsubsection{Total}

By using (68, 73, 81, 91, 92, 93), we obtain that the dominant contribution to the one-loop amplitude is given by 
- $r=\tilde{r}=s=0 \quad$ for the contributing coefficient 32

$$
\chi_{-m, 0}^{0,0}=\frac{2^{\frac{3-D}{2}}}{\pi} \sigma_{L}^{0, m} \approx \frac{N-m}{e^{\pi \sqrt{D-2} \frac{N-m}{2 \sqrt{N}}}-1}, \quad N-m \lesssim \sqrt{N}
$$

- contributions with $m \ll N$ are exponentially suppressed.

\subsection{The average mass shift}

We are able now to sum up all that we have done so far and eventually obtain the average closed string mass shift. Indeed, we can correctly individuate the asymptotic behavior of the integrals factors (41), since we know which are the dominant contributions. Once computed the integral asymptotically for large $N$, we will include the coefficient factors and perform the sum over the dominant channels in (26), which would give us the leading asymptotic behavior for the amplitude.

Thanks to the analysis in the previous sections, we have seen that the total one-loop amplitude can be subdivided in two contributions: one corresponding to massless interactions and the other to massive one. We have argued at various points (see in particular section 4.2) that the asymptotes of these two contributions are very different and that the first one is dominating.

Recall that the name "massless interactions" is a bit misleading: by this we mean that at least one of the states running in the loop is massless or a pure Kaluza-Klein/winding mode 33 .

Considering these contributions, given by $p=\tilde{p}=0$, and taking into account section 4.3.4, (41) reads

$\frac{1}{\mathcal{V}_{c}} \sum_{\left\{q_{i}\right\}} \operatorname{Re}(H)=\frac{1}{\mathcal{V}_{c}} \sum_{\left\{q_{i}\right\}} \int_{F} \frac{d^{2} \tau}{\tau_{2}^{\frac{d+1}{2}}} \int d^{2} \nu e^{-4 \pi\left|\left(N+Q^{2}\right) \frac{\nu_{2}^{2}}{\tau_{2}}+(m-N) \nu_{2}-2 q \cdot Q \nu_{2}+\left(q^{2}-\frac{n \cdot w}{2}\right) \tau_{2}\right|}$,

where we have also included the sum over winding and Kaluza-Klein modes with the volume factor for the compactified dimensions (in unit of $\alpha^{\prime}$ and neglecting factors of $2 \pi$ ):

$$
\frac{1}{\mathcal{V}_{c}} \equiv \prod_{i=1}^{d_{c}-d} \frac{1}{R_{(i)}}
$$

\footnotetext{
${ }^{32}$ This is specific for the average computation. It is easy to check, by choosing an initial string state of preference that that is not generally the case. Note that $r+\tilde{r}-s$ is related to the spin of the states.

${ }^{33}$ Which are not massless form the point of view of the extended dimensions, but are so in ten dimensions.
} 
The absolute value in the exponent takes into account the analytical continuation.

It is convenient to re-write (95) as

$$
\frac{1}{\mathcal{V}_{c}} \sum_{\left\{q_{i}\right\}} \operatorname{Re}(H)=\frac{1}{\mathcal{V}_{c}} \sum_{\left\{q_{i}\right\}} \int_{F} \frac{d^{2} \tau}{\tau_{2}^{\frac{d+1}{2}}} \int d^{2} \nu e^{-4 \pi\left|N \frac{\nu_{2}^{2}}{\tau_{2}}+(m-N) \nu_{2}+\left(\left(q_{i}-Q_{i} \frac{\nu_{2}}{\tau_{2}}\right)^{2}-\frac{n \cdot w}{2}\right) \tau_{2}\right|}
$$

From (94) and large $\sqrt{N}=\sqrt{M_{0}^{2}-Q^{2}}$, the sum over $m$ in the total amplitude (26) for the dominant contribution can be approximated by an integral, using

$$
N-m \sim y \sqrt{N}, \quad 0<y<1 .
$$

We obtain

$$
\frac{1}{\mathcal{V}_{c}} \sum_{\left\{q_{i}\right\}} \operatorname{Re}(H)=\frac{1}{\mathcal{V}_{c}} \sum_{\left\{q_{i}\right\}} \int_{F} \frac{d^{2} \tau}{\tau_{2}^{\frac{d+1}{2}}} \int d^{2} \nu e^{-4 \sqrt{N} \pi\left|\frac{\sqrt{N}}{\tau_{2}} \nu_{2}^{2}-y \nu_{2}+\left(\left(q_{i}-Q_{i} \frac{\nu_{2}}{\tau_{2}}\right)^{2}-\frac{n \cdot w}{2}\right) \frac{\tau_{2}}{\sqrt{N}}\right|} .
$$

In the limit $\sqrt{N} \gg 1$ the (Laplace) integral over $\nu_{2}$ is strongly dominated by $\nu_{2} \sim y \frac{\tau_{2}}{\sqrt{N}}$. Then, the integral over $\tau_{2}$ favors $q_{i} \sim Q_{i} \frac{y}{\sqrt{N}}$ (especially for large $\tau_{2}$, which will dominate as we will see in a moment). Since $Q \ll \sqrt{N}, M_{0}$ in the limit $34 N, M_{0} \gg 1$, then the favored channels have $q_{i} \sim 0$.

We can see that the contribution from $\tau_{2}<\sqrt{N}$ has a different asymptotic behavior. Indeed, recall that the integrand of the one-loop amplitude has in effect an expansion in terms of $\sin (\pi \nu)$ starting from the power $|\sin (\pi \nu)|^{-2}$, as dictated by the OPE in order to have the correct limit for $\nu \rightarrow 0$. But then, for a generic power $n$

$$
|\sin (\pi \nu)|^{n}=\sum_{l \geq 0}\left(\begin{array}{l}
\frac{n}{2} \\
l
\end{array}\right) \sin \left(\pi \nu_{1}\right)^{\frac{n}{2}-l}\left(\sinh \left(\pi \nu_{2}\right)\right)^{l} .
$$

When $\nu_{2} \sim 0$ asymptotically, we see that the only term that survives is the one where there are no powers of $\sinh \left(\pi \nu_{2}\right)$.

Going back to the expansion in section 3.1, we see that this shows up in the term with $m=N$. This contribution to the amplitude does not have an imaginary part, therefore its coefficient factor will have an asymptotic behavior different from those of the terms that contribute for $\tau_{2} \geq \sqrt{N}$. In fact, this contribution corresponds to the excitation of a massless state with zero momentum (in ten dimensions, see (65, 39, 401)). Such a term is related to a tadpole-like diagram. We believe that it must be subdominant because of

\footnotetext{
${ }^{34}$ Recall that $N=M_{0}^{2}-Q^{2}$.
} 
the behavior of the infrared singularity under supersymmetry or taking into account the Fischler-Susskind mechanism when supersymmetry is absent 35 . Even if this was not the case, its different origin and asymptotic behavior allows us a least to separate this contribution from the fully computable remaining one.

By rescaling $\tau_{2} \rightarrow \sqrt{N} \tau_{2} \sqrt{1-\tau_{1}^{2}}$ and taking into account the comment after formula (99), the contribution from $\tau_{2} \geq \sqrt{N}$ gives instead

$$
\begin{aligned}
\frac{1}{\mathcal{V}_{c}} \sum_{\left\{q_{i}\right\}} \operatorname{Re}(H) & \sim \frac{1}{2 \mathcal{V}_{c} \sqrt{N}} \int_{1}^{\infty} \frac{d \tau_{2}}{N^{\frac{D-3}{4}} \tau_{2}^{\frac{D}{2}-\frac{1}{2}}} \int_{-\frac{1}{2}}^{\frac{1}{2}} d \tau_{1} \frac{1}{\sqrt{1-\tau_{1} \frac{D}{2}-\frac{3}{2}}} \\
& =\frac{c}{(D-2) \mathcal{V}_{c} \sqrt{N}} N^{\frac{3-D}{4}}
\end{aligned}
$$

where $c$ is a (positive) constant (taking into account also the integration over $\left.\tau_{1}\right)$.

Stepping aside for one moment, we can see also that for contributions different from the massless ones, so that $N-m \neq y \sqrt{N}$, the integral over $\nu_{2}$ is in general asymptotically suppressed unless $\tau_{2}>N$. The not suppressed contribution goes then like $N^{\frac{2-D}{2}}$ and this tells us again that it is subdominant to the massless contribution given in (101).

To obtain the total result for the real part (26) , we sum 36 over $m$ approximating, as we said, the sum with an integral in the limit of large mass as $\sum_{m} \sim 2 \sqrt{N} \int d y$ (see (98) and we recall

$$
\chi \tilde{\chi} \sim N \frac{y^{2}}{\left(e^{\pi \frac{\sqrt{D-2}}{2} y}-1\right)^{2}}
$$

from (94) 37 . We then have to double the result to take into account massless interactions in both arcs of the loop.

We finally obtain

$$
\operatorname{Re}\left(T_{T^{2}}\right) \sim \frac{2}{D-2} c^{\prime} g_{s}^{2}\left(M_{0}^{2}-Q^{2}\right)^{1+\frac{3-D}{4}}
$$

where we have taken the compactified dimensions at radii $R_{(i)} \sim \sqrt{\alpha^{\prime}}=2$ and included the numerical factors in the (positive) constant $c^{\prime}$. This result, here

\footnotetext{
${ }^{35}$ We are aware that this is only a supposition and not a rigorous proof.

${ }^{36}$ We consider all the non-zero contributions and their relations according to (72). Among them, those asymptotically dominant are the important ones.

${ }^{37}$ Written here with a bit of sloppy notation.
} 
expressed in unit of $\alpha^{\prime}$, can be rewritten in terms of the Newton's constant in $D$ spacetime dimensions, which in this case is related to the string coupling (see[11]) by $G_{N}^{D}=(2 \pi)^{D-3} g_{s}^{2}\left(\alpha^{\prime}\right)^{\frac{D-2}{2}}$. We redefine now $g_{s}$ in order to get rid of the factor $\frac{2 c^{\prime}}{D-2}$.

Adding the result for the imaginary part from (67, 73), see also [16], we obtain 38

$$
T_{T^{2}}=g_{s}^{2}\left(M^{2}-Q^{2}\right)^{1+\frac{3-D}{4}}+i \pi g_{s}^{2} M^{2} .
$$

Note that in this last formula we have expressed the mass shift and related quantities in terms of the true mass instead than the tree-level one, which is probably even a more accurate estimate.

We can now determine the average mass shift for closed string states. Since we use a mostly plus metric, the field propagator for an unstable state i. 39

$$
G(p) \sim \frac{Z}{p^{2}+M^{2}-i M \Gamma},
$$

where $\Gamma$ is the total decay. Indeed the time dependence of the propagator shows the expected exponential decay:

$$
\begin{aligned}
G(\vec{p}, t) & =\int \frac{d p^{0}}{2 \pi} e^{-i p^{0} t} G(p) \sim \frac{Z}{\sqrt{p^{2}+M^{2}-i M \Gamma}} e^{-i \sqrt{p^{2}+M^{2}-i M \Gamma}} \\
& \sim \frac{Z}{\sqrt{p^{2}+M^{2}-i M \Gamma}} e^{-i E_{\vec{p}} t-\frac{M}{E_{\vec{p}}} \Gamma t} .
\end{aligned}
$$

Therefore, since it is $\Gamma=M^{-1} \operatorname{Im}\left(T_{T^{2}}\right)$, it must be

$$
p^{2}+M_{0}^{2}-T_{T^{2}}=p^{2}+M_{0}^{2}-\operatorname{Re}\left(T_{T^{2}}\right)-i \operatorname{Im}\left(T_{T^{2}}\right)=p^{2}+M^{2}-i M \Gamma,
$$

so that the average squared string mass shift at mass level $N$ is

$$
\Delta M^{2} \equiv M^{2}-M_{0}^{2}=-\operatorname{Re}\left(T_{T^{2}}\right)=-g_{s}^{2}\left(M^{2}-Q^{2}\right)^{1+\frac{3-D}{4}} .
$$

\section{Discussion and Conclusion}

In this work we have presented a detailed investigation of (closed) string one-loop amplitudes in string theory, in particular dealing with the average mass shift for states at a given squared mass and Neveu-Schwarz charges. Our algorithm is based only on well-defined string amplitudes and the exploitation of symmetries and unitarity properties of the torus amplitudes.

\footnotetext{
${ }^{38}$ We write here only the massless contribution to the imaginary part.

${ }^{39} \mathrm{We}$ are not interested in the details of the numerator.
} 
We have been able to argue and show at various moments (sections 3.2.2, 4.2, and after formula (101)) that the dominant contribution to the decay channel is given by long-range interaction (in fact gravitational ones).

We are aware, though, that our arguments are evidential, but not an exhaustive mathematical proof. However they strengthen the physical intuition that gravitational interactions (which grows with the mass) are the dominant one for very massive states. Furthermore, our results, coming form a direct string theory computation, clarify and correct previous estimates of the string self-energy, obtained so far only in approximated models which assumed the predominance of gravitational interactions from the outset.

In the limit of large mass, we find that the average squared mass shift for states at a given mass is

$$
\Delta M^{2}=-g_{s}^{2}\left(M^{2}-Q^{2}\right)^{1+\frac{3-D}{4}} .
$$

The interaction becomes important when (consider the case $Q^{2}=0$ )

$$
g_{s} \sim M^{\frac{d-6}{4}}
$$

which is the expected behavior for this kind of interactions (see formulas 3.3, 3.4 in [2]).

Discussing (109) more in details, we distinguish two contributions to this result: the first one $\left(M^{2}-Q^{2}\right)$ comes from the coefficients (102) and therefore the coupling, the second factor $\left(\left(M^{2}-Q^{2}\right)^{\frac{3-D}{4}}\right)$ comes from the integration over $\tau_{2}$.

Note that $\tau_{2}$ is linked to the range of the interactions. Indeed if we consider the string formula (44) in the operator formalism (see [18]), it is related to the internal loop momentum $k$ by $e^{-\tau_{2} k^{2}}$. We expect it, therefore, to be related to the squared range $L^{2}$ of the interactions, and looking at (95), 109), we find that this is $L^{2} \sim \sqrt{N}=\sqrt{M^{2}-Q^{2}}$.

This result can be understood in a simple way. We observe that a string at large mass is well-represented at tree-level by a random-walk of size $L^{2} \sim \sqrt{N}$ ([1]) and assume a Newton potential acting between two points on the string 40 $\int \frac{d r^{\prime}}{L} \int \frac{d r}{L} r^{D-3}$. We expect that the integration will have an upper bound given by the size $L$ of the string, so that the contribution is $\sim L^{3-D}$, which is the second factor we find.

In performing the Newton potential calculation we have neglected the contribution that arises from the small distances along the string (lower end of the integral), for which a classical picture is not suited: it is only the quantum computation that can give us the precise result.

\footnotetext{
${ }^{40}$ The factors $L^{-1}$ represent an averaging since it is irrelevant where along the walk the integrations start.
} 
An interesting picture of one-loop corrections to the string in non - supersymmetric configurations arises: the dominant interactions responsible for the corrections are of long-range type (namely gravitational) and it appears that perturbations theory is generally reliable on the whole spectrum of massive string states in any dimensions 41 .

\section{Acknowledgments}

I would like to thank Igor Pesando and Paolo di Vecchia for the many conversations regarding this project. I am especially grateful to Bo Sundborg for his comments on the results of this work.

\section{A Computation of the residue $\mathcal{R}_{m}^{r, s}$}

Here we compute the residue $\mathcal{R}_{m}^{r, s}$ involved in the derivation of the finite difference equations for the one-loop amplitude series coefficients, see (47). Recall its definition

$$
\mathcal{R}_{m}^{r, s}=\frac{1}{2 \pi i} \oint_{\mathcal{C}_{1}} \frac{d v}{v} v^{m+N} \mathcal{F}^{r, s}(v, w),
$$

where $\mathcal{C}_{1}$ is a circuit around $v=1$ (see figure 3). In order to find $\mathcal{R}_{m}^{r, s}$ we need to expand $\mathcal{F}^{r, s}(v, w)$ around $v=1$, that is $\nu=0$ (recall $v \equiv e^{2 \pi i \nu}$ ).

Recall the definition (20) of $\mathcal{F}^{r, s}(v, w)$, here reproduced for the reader convenience

$$
\left(2 \pi i \frac{\theta_{1}(\nu, \tau)}{\theta_{1}^{\prime}(0, \tau)}\right)^{2 N} \mathcal{D}_{s}^{r}\left(\partial^{2} \log \theta_{1}, \partial^{3} \log \theta_{1}, \ldots\right) \mathcal{X}_{\phi}(\nu, \tau) \equiv \mathcal{F}^{r, s}(\nu, \tau) .
$$

We know that

$$
\begin{aligned}
\frac{\theta_{1}(\nu, \tau)}{\theta_{1}^{\prime}(0, \tau)} & \sim \frac{\nu}{2}-\frac{\pi^{2}}{12} \nu^{3} \\
\partial_{\nu}^{n} \log \theta_{1} & \sim \frac{a_{n}}{\nu^{n}}(1+O(\nu))
\end{aligned}
$$

for some constant $a_{n}$ depending on $n$.

\footnotetext{
${ }^{41}$ That is to say, given a certain $g_{s}<1$, set by the dilaton vacuum expectation value, the correction (109) is never larger than the tree-level value for any mass. Our result does not exclude that particular sets of states, not representing significant portion of the string spectrum (sets of "measure zero") and therefore not affecting the average, could have larger corrections and therefore not be suited for a perturbative treatment, such as possibly the leading Regge trajectory states of [8].
} 
On the other hand, the OPE among physical vertex operators 42 dictates the most singular term to be (left-moving part only)

$$
V_{\phi}^{L}(1) V_{\phi}^{L}(v) \sim \frac{1}{\nu^{2}}
$$

and since the leading singularity in the correlator is given indeed by the OPE computation, we find

$$
\mathcal{D}_{s}^{r}\left(\partial^{2} \log \theta_{1}, \partial^{3} \log \theta_{1}, \ldots\right) \mathcal{X}_{\phi}(\nu, \tau) \sim \frac{c}{\nu^{2 N+2-2 r}} .
$$

This is straightforward to understand by observing formulas (14, 18, 19) and realizing that the most singular term in the expansion (18) for $\mathcal{P}(W, \Omega, \widetilde{\Omega}, \ldots)$ comes from the term $\mathcal{D}_{0}^{0}\left(\partial^{2} \log \theta_{1}, \partial^{3} \log \theta_{1}, \ldots\right) \mathcal{D}_{0}^{0}\left(\bar{\partial}^{2} \log \bar{\theta}_{1}, \bar{\partial}^{3} \log \bar{\theta}_{1}, \ldots\right)$ because of (114). Terms with $r, \tilde{r} \neq 0$ will have less singular behavior for $\nu \rightarrow 0$ because they contain $r$ double derivative of $\log \theta_{1}$ less than the case $r=\tilde{r}=0$ according to (14, 19),

Therefore, from (111, 113, 116) we obtain

$$
\begin{aligned}
\mathcal{R}_{m}^{0,0} & =\frac{1}{2 \pi i} \oint_{\mathcal{C}_{1}} \frac{d v}{v} v^{m+N} \mathcal{F}^{0,0}(v, w) \\
& =\frac{1}{2 \pi i} \oint \frac{d \nu}{\nu} e^{2 \pi(m+N) i \nu} \frac{A^{0} \nu^{2 N}}{\nu^{2 N+2}} Z_{T^{2}}=0 .
\end{aligned}
$$

for a supersymmetric background ( $Z_{T^{2}}$ is the relevant partition function). Note that in a non -supersymetric case we would have found $\mathcal{R}_{m}^{0,0}=A^{0}(m+$ $N)$, where $A^{0}$ is a constant that for normalized states is $A^{0}=1$ because of the OPE (115).

Similarly we find

$$
\begin{aligned}
\mathcal{R}_{m}^{r \neq 0, s} & =\frac{1}{2 \pi i} \oint_{\mathcal{C}_{1}} \frac{d v}{v} v^{m+N} \mathcal{F}^{r, s}(v, w) \\
& =\frac{1}{2 \pi i} \oint \frac{d \nu}{\nu} e^{2 \pi(m+N) i \nu} \frac{A^{r, s} \nu^{2 N}}{\nu^{2 N+2-2 r}}=0, \quad \forall r \geq 1 .
\end{aligned}
$$

We have then obtained (49).

The fact that poles at $v=1$ are not present in supersymmetric backgrounds also clarifies the results in (172). Indeed, the fact that massless channels with $m<-2 N, p=\tilde{p}=0$ do not contribute to the one-loop amplitude, having a zero coefficient factor could seem at first sight perplexing. But it is simple to understand it once one realizes that the amplitude has an

\footnotetext{
${ }^{42}$ Here we assume normalized states.
} 
expansion in powers of $(1-v)$ and only positive powers can occur, in order not to have poles at $v=1$. By looking at (44), it is possible to see that the maximum power to appear in the limit $w \rightarrow 0$ is $(1-v)^{2(N-1)}$.

The same should occur to massless contributions having $m=p \neq 0, \tilde{p}=$ $p+\sum_{i} n_{i} w_{i}$. We can verify it by using the formulas for the theta functions in appendix C. By changing variables to $x_{1}=e^{2 \pi i(\tau-\nu)}, x_{2}=e^{2 \pi i \nu}$, it is straightforward to see that those contributions are accounted for by the term of order $x_{2}^{0}$ in (4) which can be written as a series expansion in $1-w$ with no negative powers and maximum power $(1-w)^{2(N-1)}$.

\section{B Arguments for suppression due to averag- ing}

We show here how simple arguments can make us understand why averaging cannot individuate a preferred contribution channel to the one-loop coefficients. We concentrate on those contributions to the amplitude which have a non-zero imaginary part. In those cases the coefficient of expansion (26) are related to the decay rates as in (68). Let us concentrate on the latter, then.

Recall

$$
\Gamma_{i}=g_{s}^{2} \mathcal{P} \sigma_{L} \times \sigma_{R},
$$

which has been defined in section 4.2 and by formula (63) which we re-write here for the reader's convenience:

$$
\sigma_{L}^{\aleph, N^{\prime}}=\frac{1}{\sqrt{G_{c}(N)}} \sum_{\zeta_{\left.\right|_{\aleph}}} \oint \frac{d z}{z^{N+1}} \oint \frac{d z^{\prime}}{z^{\prime}} z^{\prime N-N^{\prime}} \operatorname{tr}\left[V_{\zeta, L}^{\dagger}(1) V_{\zeta, L}\left(z^{\prime}\right) z^{\hat{N}_{L}}\right] .
$$

As we said in section 4.2, apparently the suppressing factor $G_{c}(N)^{-1} \sim$ $e^{-2 \pi \sqrt{d-1} \sqrt{N}}$ in (119) could lead to a kinetic suppression of many contributions.

In order to investigate when this is the case, let us further study the quantity $\sigma_{R,(L)}$. It is straightforward to realize that one gets

$$
\begin{aligned}
\operatorname{tr}\left[V_{\zeta, R}^{\dagger}(1) V_{\zeta, R}(v) w^{\hat{N}}\right] & =f(w)^{2-D} F(v, w) \\
f(w) & =\prod_{n=1}^{\infty}\left(1-w^{n}\right)
\end{aligned}
$$

where the factor $f(w)^{2-D}$ comes from the contractions among the (bosonic part of the) states running in the trace (alternatively one reaches the same conclusion by realizing that (120) is a one-loop amplitude projected down 
to some definite mass levels and without interaction over zero modes of the fields). This factor is where one expects the large degeneracies to come from.

$F(v, w)$ is a function of $\Omega$ defined in (5) and its higher derivatives (and not of $W$ as instead $P$ in (4) ) .

By looking at the definition of $\Omega$ (and therefore of its derivatives), we see that the term of order $v^{N^{\prime}-N}$ picked up by the $v$ loop integration in (63) is accompanied (generally) by a factor $w^{\left(N-N^{\prime}\right) \ell}, \ell \geq 1$. Therefore the final loop integral becomes $\sim \oint d w w^{N(\ell-1)-N^{\prime}-1}$ and we see that we obtain the largest contribution $\sim e^{\pi \sqrt{d-1} \sqrt{N^{\prime}}}$ for $\ell=1$.

On the other end we have to sum over all possible $|\zeta\rangle$ at mass level $\aleph \sim N-N^{\prime}$ with degeneracy $e^{\pi \sqrt{d-1} \sqrt{N-N^{\prime}}}$ and this implies a total result of $e^{\pi \sqrt{d-1}\left(\sqrt{N-N^{\prime}}+\sqrt{N^{\prime}}\right)}$. Similar arguments apply to $\sigma_{L}$. The product of these two results compensates the suppressing factor $G_{c}(N)^{-1} \sim e^{-2 \pi \sqrt{d-1} \sqrt{N}}$ in (119).

\section{Theta functions}

Our conventions for the theta functions are

$$
\begin{gathered}
\theta_{1}=i \sum_{n=0}^{\infty}(-1)^{n} w^{\frac{\left(n+\frac{1}{2}\right)^{2}}{2}}\left(v^{n+\frac{1}{2}}-v^{-n-\frac{1}{2}}\right) \quad \theta_{2}=\sum_{n=0}^{\infty} w^{\frac{\left(n+\frac{1}{2}\right)^{2}}{2}}\left(v^{n+\frac{1}{2}}+v^{-n-\frac{1}{2}}\right) \\
\theta_{3}=1+\sum_{n=1}^{\infty} w^{\frac{n^{2}}{2}}\left(v^{n}+v^{-n}\right) \quad \theta_{4}=1+\sum_{n=1}^{\infty}(-1)^{n} w^{\frac{n^{2}}{2}}\left(v^{n}+v^{-n}\right) .
\end{gathered}
$$

\section{References}

[1] D. Chialva, "Self-interacting fundamental strings and black holes", arXiv:0903.3977 [hep-th].

[2] G. T. Horowitz and J. Polchinski, Phys. Rev. D 57 (1998) 2557 arXiv:hep-th/9707170.

[3] T. Damour and G. Veneziano, Nucl. Phys. B 568 (2000) 93 arXiv:hep-th/9907030.

[4] R. B. Wilkinson, N. Turok and D. Mitchell, Nucl. Phys. B 332 (1990) 131.

[5] D. Mitchell, N. Turok, R. Wilkinson and P. Jetzer, Nucl. Phys. B 315 (1989) 1 [Erratum-ibid. B 322 (1989) 628]. 
[6] D. Chialva, R. Iengo and J. G. Russo, JHEP 0312 (2003) 014 arXiv:hep-th/0310283]. D. Chialva and R. Iengo, JHEP 0407 (2004) 054 [arXiv:hep-th/0406271]. H. Okada and A. Tsuchiya, Phys. Lett. B 232 (1989) 91. M. Gutperle and D. Krym, Phys. Rev. D 74 (2006) 086007 arXiv:hep-th/0607220.

[7] N. Seiberg, Phys. Lett. B 187 (1987) 56. A. Sen, Nucl. Phys. B 304 (1988) 403. J. Polchinski, Nucl. Phys. B 307 (1988) 61.

[8] B. Sundborg, Nucl. Phys. B 319 (1989) 415.

[9] A. Liccardo, F. Pezzella and R. Marotta, Mod. Phys. Lett. A 14 (1999) 799 arXiv:hep-th/9903027.

[10] S. Hwang, Phys. Lett. B 276 (1992) 451 arXiv:hep-th/9110039]. J. M. Evans, M. R. Gaberdiel and M. J. Perry, Nucl. Phys. B 535 (1998) 152 arXiv:hep-th/9806024]. N. Lambert, H. Liu and J. M. Maldacena, JHEP 0703 (2007) 014 arXiv:hep-th/0303139|. N. Jokela, E. Keski-Vakkuri and J. Majumder, Phys. Rev. D 73 (2006) 046007 [arXiv:hep-th/0510205].

[11] J. Polchinski, "String theory. Vol. 1: An introduction to the bosonic string," "String theory. Vol. 2: An introduction to the superstring," Cambridge, UK: Univ. Pr. (1998).

[12] W. Fischler and L. Susskind, Phys. Lett. B 171 (1986) 383; W. Fischler and L. Susskind, Phys. Lett. B 173 (1986) 262.

[13] L. Rastelli and B. Zwiebach, JHEP 0801 (2008) 018 arXiv:0708.2591 [hep-th]].

[14] J. L. Manes, Nucl. Phys. B 621, 37 (2002) arXiv:hep-th/0109196].

[15] D. Amati and J. G. Russo, Phys. Lett. B 454 (1999) 207 arXiv:hep-th/9901092].

[16] D. Chialva, R. Iengo and J. G. Russo, JHEP 0501, 001 (2005) arXiv:hep-th/0410152].

[17] J. J. Atick and A. Sen, Nucl. Phys. B 286 (1987) 189.

[18] M. B. Green, J. H. Schwarz and E. Witten, "Superstring Theory. Vol. 2: Loop Amplitudes, Anomalies And Phenomenology," Cambridge, Uk: Univ. Pr. (1987) 596 P. (Cambridge Monographs On Mathematical Physics). 